\title{
Real secondary index theory
}

\author{
ULRICH BUNKE \\ THOMAS SCHICK
}

\begin{abstract}
In this paper, we study the family index of a family of spin manifolds. In particular, we discuss to what extent the real index (of the Dirac operator of the real spinor bundle if the fiber dimension is divisible by 8 ) which can be defined in this case contains extra information over the complex index (the index of its complexification). We study this question under the additional assumption that the complex index vanishes on the $k-$ skeleton of $B$. In this case, we define new analytical invariants $\widehat{c}_{k} \in H^{k-1}(B ; \mathbb{R} / \mathbb{Z})$, certain secondary invariants.
\end{abstract}

We give interesting nontrivial examples. We then describe this invariant in terms of known topological characteristic classes.

$57 \mathrm{R} 20$

\section{Introduction}

1.0.1 The index of a family of Fredholm operators parametrized by a space $B$ is an element in the $K$-theory $K^{*}(B)$ of this parameter space. If the base is in fact a smooth compact manifold without boundary, and this family is a family of fiberwise generalized Dirac operators on a smooth fiber bundle over $B$, then after adding some further geometric structures in order to define the Bismut super connection we can do local index theory in the sense of Berline, Getzler and Vergne [4]. Let us denote by $\mathcal{E}$ the family with this collection of geometric structures, by $D(\mathcal{E})$ the family of Dirac operators, and by index $(\mathcal{E})$ the index of this family. Local index theory provides a closed form $\Omega(\mathcal{E})$ on $B$ (see Definition 2.1) which represents a cohomology class $[\Omega(\mathcal{E})] \in H^{*}(B, \mathbb{R})$. The local index theorem states that

$$
\operatorname{ch}^{\mathbb{R}}(\operatorname{index}(\mathcal{E}))=[\Omega(\mathcal{E})] .
$$

1.0.2 The focus of the present paper is not a generalization of this type of result. Let us illustrate the philosophy of the present paper in the case above. We start with local index theory and produce the even form $\Omega(\mathcal{E})$. We then observe that this form is closed and therefore represents a cohomology class $[\Omega(\mathcal{E})] \in H^{\mathrm{ev}}(B, \mathbb{R})$. We observe that this class in fact only depends on $\operatorname{index}(\mathcal{E}) \in K^{0}(B)$. The classifying space of the 
functor $K^{0}$ is $B U \times \mathbb{Z}$. By naturality we conclude that there must be a universal class $\mathbf{c h}_{\text {univ }}^{\mathbb{R}} \in H^{\text {ev }}(B U \times \mathbb{Z}, \mathbb{R})$ such that $[\Omega(\mathcal{E})]=f^{*} \mathbf{c h}_{\text {univ }}^{\mathbb{R}}$, if $f: B \rightarrow B U \times \mathbb{Z}$ classifies index $(\mathcal{E})$. We know that $H^{\mathrm{ev}}(B U, \mathbb{R})$ is a polynomial ring in generators $c_{2}^{\mathbb{R}}, c_{4}^{\mathbb{R}}, \ldots$ Then we finally look for a formula which expresses $\mathbf{c h}_{\text {univ }}^{\mathbb{R}}$ in terms of these generators, this way obtaining the Chern character. Of course this is a well known possible way toward the family index formula.

1.0.3 In fact the real subject of the present paper is a secondary version of this approach. Let $K_{k}^{*}(B)$ denote the $k$ 'th step of the Atiyah-Hirzebruch filtration of $K$-theory (see 2.1.6), ie the subgroup of classes which vanish when restricted to the $(k-1)$-skeleton of $B$ (so that $K_{0}^{*}(B)=K(B)$ ). Under the assumption that $\operatorname{index}(\mathcal{E}) \in K_{k}^{*}(B)$, the Chern class $c_{k}(\operatorname{index}(\mathcal{E})) \in H^{k}(B, \mathbb{Z})$ (note that we use a nonstandard notation where the subscript is equal to the degree) admits a natural lift to smooth Deligne cohomology $\widehat{c}_{k}(\mathcal{E}) \in H_{\text {Del }}^{k}(B)$ (see 2.1.8 and 2.1.17). This lift is a differential-geometric (or even global-analytic) invariant which varies continuously with the geometry. In particular it has a curvature $\omega\left(\widehat{c}_{k}(\mathcal{E})\right) \in \mathcal{A}^{k}(B)$, which can be expressed through $\Omega(\mathcal{E})$.

1.0.4 We rigidify the situation be imposing additional geometric constraints. We in fact assume that the family of Dirac operators $D(\mathcal{E})$ is a family of twisted Dirac operators on a family of Spin-manifolds, and that the twisting bundle is a real bundle. Let $n$ be the fiber-dimension of this family. It follows from the presence of the real structure that, if $k+n \equiv 2(4)$ then the class $\widehat{c}_{k}(\mathcal{E})$ is flat (see 2.1.19). This means that $\omega\left(\widehat{c}_{k}(\mathcal{E})\right)=0$. Since any two geometric structures can be connected by a path we can now conclude that under this assumptions $\widehat{c}_{k}(\mathcal{E})$ is a differential-topological invariant. In Section 3.3 we give some nontrivial examples.

1.0.5 Note that the flat part of $H_{\mathrm{Del}}^{k}(B)$ can be identified with $H^{k-1}(B, \mathbb{R} / \mathbb{Z})$. Thus, given a family of $n$-dimensional spin manifolds and a real twisting bundle such that $\operatorname{index}(\mathcal{E}) \in K_{k}^{*}(B)$ and $k+n \equiv 2(\bmod 4)$ we have defined a class $\widehat{c}_{k}(\mathcal{E}) \in$ $H^{k-1}(B, \mathbb{R} / \mathbb{Z})$. This class is natural under pullback.

The index of the family $D(\mathcal{E})$ has a real refinement $\operatorname{index}_{\mathbb{R}}(\mathcal{E}) \in K O^{-n}(B)$ (see Section 2.2). We prove that in fact $\widehat{c}_{k}(\mathcal{E})$ only depends on $\operatorname{index}_{\mathbb{R}}(\mathcal{E})$ and that $2 \widehat{c}_{k}(\mathcal{E})=0$ (see Proposition 3.2 and Proposition 3.5).

Let $U_{l}^{-n}(B) \subset K O^{-n}(B)$ be the subset of classes which after complexification belong to $K_{l}^{-n}(B)$. What we have constructed so far is a natural transformation

$$
d_{B, 4 k+1-n}^{-n}: U_{4 k+2-n}^{-n}(B) \rightarrow H^{4 k+1-n}(B, \mathbb{R} / \mathbb{Z})
$$

such that $d_{B, 4 k+1-n}^{-n}\left(\operatorname{index}_{\mathbb{R}}(\mathcal{E})\right)=\widehat{c}_{4 k+2}(\mathcal{E})$. 
1.0.6 The universal situation is given by the fiber sequence

$$
\Omega^{n}(U / O) \stackrel{\Omega^{n} i}{\rightarrow} \Omega^{n}(B O \times \mathbb{Z}) \rightarrow \Omega^{n}(B U \times \mathbb{Z}) .
$$

It is obtained by application of the functor $\Omega^{n}$ to the fibration $U / O \stackrel{i}{\rightarrow} B O \rightarrow B U$, where we construct $B O=E U / O$ and thus obtain the inclusion $i: U / O \rightarrow B O$. A class $x \in K O^{-n}(B)$ is represented by a map $f: B \rightarrow \Omega^{n}(B O \times \mathbb{Z})$. If $x$ belongs to $U_{\infty}^{-n}(B)$, then this map factors up to homotopy through a map $g: B \rightarrow \Omega^{n}(U / O)$. Thus there must be a universal class

$$
\bar{d}_{4 k+1-n} \in H^{4 k+1-n}\left(\Omega^{n}(U / O), \mathbb{R} / \mathbb{Z}\right)
$$

such that $g^{*} \bar{d}_{4 k+1-n}=d_{B, 4 k+1-n}^{-n}(x)$. Note that this universal class has the special property that $g^{*} \bar{d}_{4 k+1-n}$ only depends on the homotopy class of the composition $\Omega^{n} i \circ g$.

1.0.7 The main purpose of the present paper is the calculation of the universal class $\bar{d}_{4 k+1-n}$ in terms of the classically known generators of the cohomology of $\Omega^{n}(U / O)$. The result is presented in Theorem 4.10.

1.0.8 If $n=2,3,4,5$ or $n=1, k>0$, then $\bar{d}_{4 k+1-n}=0$. If $n=0$ or $n=1, k=0$ then the class $d_{B, 4 k+1-n}^{-n}(x)$ is a classical characteristic class of $x$ (ie it can be expressed in terms of the dimension and Stiefel-Whitney classes). If $n=7$, in principle it is also a well-known characteristic class (albeit for $\mathrm{KO}^{7}$ which is not considered much), ie pulls back from the classifying space $U / O$.

The dimension $n=6$ is interesting since in this case the classes are definitely not just classical characteristic classes of $x$.

See Section 4.5 for all that.

1.0.9 For the convenience of the reader we have added two appendixes. In the first we recall (with proof) some material about transgression.

In the second we recall the results of Cartan [8] about the cohomology of the spaces $\Omega^{n}(U / O)$ and about the action of various maps and transgressions on this cohomology.

\section{Real local index theory}

\subsection{Chern classes of geometric families in Deligne cohomology}

2.1.1 We consider a fiber bundle $\pi: E \rightarrow B$ with closed $n$-dimensional fibers. We assume that the vertical bundle $T^{v} \pi:=\operatorname{ker}(d \pi)$ is oriented and equipped with a spin 
structure. We choose a vertical Riemannian metric $g^{T^{v} \pi}$ and a horizontal distribution $T^{h} \pi$, ie a complement of $T^{v} \pi$ in $T E$. Finally, we let $\mathbf{W}:=\left(W, \nabla^{W}, h^{W}\right)$ be an auxiliary complex vector bundle with hermitian metric and metric connection. The data described so far make up a geometric family $\mathcal{E}$ over $B$.

2.1.2 We assume that $\mathbf{W}$ admits a real structure $Q \in \operatorname{End}\left(W_{\mid \mathbb{R}}\right)$ which is compatible with the connection and the metric. Then $\mathbf{W}$ is the complexification of a real bundle $\mathbf{W}_{\mathbb{R}}=\left(W_{\mathbb{R}}, \nabla^{W_{\mathbb{R}}}, h^{W_{\mathbb{R}}}\right)$. The latter can be identified with the +1 -eigenbundle of $Q$.

2.1.3 The data which we compressed in the notion of a geometric family induces a family of elliptic operators $D(\mathcal{E})$ over $B$. Indeed, for $b \in B$ the operator $D(\mathcal{E})(b)$ is the spin Dirac operator of the Riemannian spin manifold $E_{b}:=\pi^{-1}(b)$ twisted by the bundle $\mathbf{W}_{\mid E_{b}}$. The family index of $D(\mathcal{E})$ is the element index $(\mathcal{E}) \in K^{-n}(B)$.

2.1.4 For $k \in \mathbb{N}_{0}$ we introduce a natural transformation $c_{k}: K^{n}(B) \rightarrow H^{k}(B, \mathbb{Z})$ given by the Chern class. In order to have a uniform notation in the even and odd dimensional case we use a notation which differs from the conventional one. So if $n$ is even, then we set $c_{2 k}:=\mathbf{c}_{k}$ and $c_{2 k+1}:=0$, where $\mathbf{c}_{k}: K^{0}(B) \rightarrow H^{2 k}(B, \mathbb{Z})$ is the Chern class in the usual notation. If $n$ is odd, then we set $c_{2 k}=0$ and define $c_{2 k+1}$ such that the following diagram is commutative

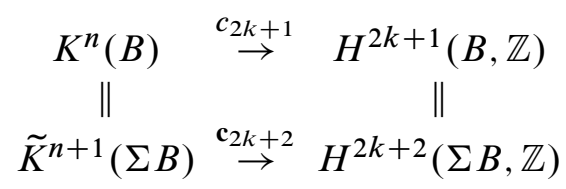

where $\widetilde{K}$ denotes the reduced $K$-theory and the vertical isomorphisms are the natural suspension isomorphisms.

2.1.5 The Chern character is a natural transformation

$$
\text { ch: } K^{n}(B) \rightarrow \bigoplus_{k \equiv n(2)} H^{k}(B, \mathbb{Q}) \text {. }
$$

Here again for the even part ch: $K^{2 n}(B) \rightarrow \bigoplus_{k \equiv 0(2)} H^{k}(B, \mathbb{Q})$ we use the usual convention, while the odd part is defined such that the following diagram is commutative:

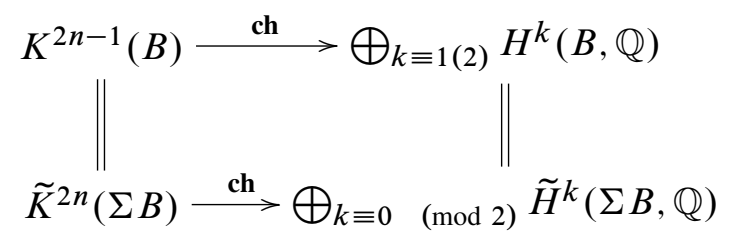

For $k \in \mathbb{N}_{0}$ let $\operatorname{ch}_{k}: K^{*}(B) \rightarrow H^{k}(B, \mathbb{Q})$ denote the corresponding component. 
2.1.6 The ring $K^{*}(B)$ has a natural decreasing filtration, the Atiyah-Hirzebruch filtration [1],

$$
\cdots \subset K_{k+1}^{*}(B) \subset K_{k}^{*}(B) \subset \cdots \subset K_{0}^{*}(B)=K^{*}(B) .
$$

Recall that $x \in K_{k}^{*}(B)$ iff $f^{*} x=0$ for any $(k-1)$-dimensional CW-complex $X$ and continuous map $f: X \rightarrow B$.

2.1.7 Fix now $k \in \mathbb{N}_{0}$ and define $m \in \mathbb{N}$ such that $k=2 m$ or $k=2 m-1$. If $x \in K_{k}^{*}(B)$, then we have

$$
c_{k}(x)_{\mathbb{Q}}=(-1)^{m-1}(m-1) ! \mathbf{c h}_{k}(x)
$$

where $c_{k}(x)_{\mathbb{Q}} \in H^{k}(B, \mathbb{Q})$ is the natural image of $c_{k}(x)$ in cohomology with rational coefficients.

2.1.8 Let $H_{\text {Del }}^{*}(B)$ denote the smooth Deligne cohomology of $B$. In the present paper we use its description in terms of differential characters given by CheegerSimons [9]. Let $Z^{k-1}$ be the group of smooth singular chains on $B$. A class $\hat{x} \in$ $H_{\text {Del }}^{k}(B)$ is a homomorphism $\hat{x}: Z^{k-1} \rightarrow \mathbb{R} / \mathbb{Z}$ such that there exists a differential form $\omega(\hat{x}) \in \mathcal{A}^{k}(B)$ with the property that for any smooth singular $k$-chain $C$ we have $\hat{x}(\partial C)=\left[\int_{C} \omega(\hat{x})\right]$, where $[r] \in \mathbb{R} / \mathbb{Z}$ denotes the class of $r \in \mathbb{R}$. Note that $\omega(\hat{x})$ is uniquely determined by $\hat{x}$. It is called the curvature of $\hat{x}$. It is necessarily closed and has integral periods.

The association $B \mapsto H_{\text {Del }}^{*}(B)$ is a contravariant functor from smooth manifolds and smooth maps to graded abelian groups. There is a natural exact sequence

$$
H^{k-1}(B, \mathbb{Z}) \rightarrow \mathcal{A}^{k-1}(B) / \operatorname{im}(d) \stackrel{\widehat{a}}{\rightarrow} H_{\mathrm{Del}}^{k}(B) \stackrel{v}{\rightarrow} H^{k}(B, \mathbb{Z}) \rightarrow 0
$$

where $a$ is given by

$$
\widehat{a}(\beta)(Z)=\left[\int_{Z} \alpha\right], \quad \beta \in \mathcal{A}^{k-1}(B) .
$$

Note that $\omega(\hat{a}(\beta))=d \beta$. The map $v$ has the following description. Let $\hat{x} \in H_{\text {Del }}^{k}(B)$. We choose a smooth $\mathbb{R}$-valued $(k-1)$-cochain $T$ such that $T_{\mid Z^{k-1}}=\hat{x}$. This is possible since $\mathbb{R}$ is divisible. Then we have $d T=\omega-c$ for some $\mathbb{Z}$-valued $k-$ cochain $c$. It follows that $c$ is closed, and we set $v(\hat{x}):=[c]$. For details we refer to Cheeger-Simons [9].

2.1.9 A complex vector bundle $W \rightarrow B$ represents an element $[W] \in K^{0}(B)$. Assume that $W$ comes with a hermitian metric $h^{W}$ and metric connection $\nabla^{W}$. We set $\mathbf{W}:=$ $\left(W, h^{W}, \nabla^{W}\right)$. For $k \in \mathbb{N}_{0}$, Cheeger-Simons [9] constructed a natural lift $\widehat{c}_{2 k}(\mathbf{W}) \in$ 
$H_{\text {Del }}^{2 k}(B)$ of $c_{2 k}([W])$ such that $v\left(\hat{c}_{2 k}(\mathbf{W})\right)=c_{2 k}([W])$ and $\omega\left(\hat{c}_{2 k}(\mathbf{W})\right) \in \mathcal{A}^{2 k}(B)$ is the Chern-Weil representative of $c_{2 k}([W])_{\mathbb{R}}$ associated to the connection $\nabla^{W}$.

2.1.10 The bundle $\mathbf{W}$ can be considered as a geometric family $\mathcal{W}$ over $B$ with zero-dimensional fiber in a natural way. In this case we have $\operatorname{index}(\mathcal{W})=[W]$.

Therefore we can consider the geometric family $\mathcal{E}$ over $B$ as a generalization of a hermitian vector bundle with connection over $B$. It is now an obvious question whether one can define a natural lift $\widehat{c}_{k}(\mathcal{E}) \in H_{\text {Del }}^{k}(B)$ of $c_{k}(\operatorname{index}(\mathcal{E}))$.

2.1.11 The geometric data associated with the geometric family $\mathcal{E}$ induces a connection $\nabla^{T^{v} \pi}$ on the vertical bundle in a natural way. In fact, if we choose for a moment a Riemannian metric $g^{T B}$ on the base, then we can define a Riemannian metric $g^{T E}$ on the total space $E$ as the orthogonal sum of the vertical metric $g^{T^{v}} \pi$ and the metric $g^{T^{h} \pi}$ on the horizontal bundle obtained by lifting $g^{T B}$. Then $\nabla^{T^{v}} \pi$ is the projection of the Levi-Civita connection of $g^{T E}$ to the vertical bundle. This connection does not depend on the choice of $g^{T B}$. We refer to [4] for details. By $\widehat{A}\left(\nabla^{T^{v} \pi}\right) \in \mathcal{A}^{*}(E)$ we denote the Chern-Weil representative of the $\hat{A}$-class of $T^{v} \pi$. Furthermore, let $\operatorname{ch}\left(\nabla^{W}\right) \in \mathcal{A}(E)$ be the Chern-Weil representative of the Chern character of $W$.

Definition 2.1 The local index form $\Omega(\mathcal{E}) \in \mathcal{A}(B)$ of the geometric family $\mathcal{E}$ is defined by

$$
\Omega(\mathcal{E}):=\int_{E / B} \hat{A}\left(\nabla^{T^{v} \pi}\right) \operatorname{ch}\left(\nabla^{W}\right)
$$

2.1.12 The Atiyah-Singer index theorem for families states that

$$
\operatorname{ch}(\operatorname{index}(\mathcal{E}))_{\mathbb{R}}=[\Omega(\mathcal{E})],
$$

where $[\omega] \in H^{*}(B, \mathbb{R})$ denotes the class represented by the closed form $\omega$. Here we once and for all fix the isomorphism between de Rham cohomology $H_{d R}^{*}(M)$ and singular cohomology $H^{*}(B, \mathbb{R})$ which is induced by the integration map. This means that the value of the class $[\omega]$ on the cycle $Z$ is given by $\int_{Z} \omega$.

2.1.13 The form $\Omega(\mathcal{E})$ plays the role of the Chern-Weil representative of the Chern character of an index bundle with connection $\nabla^{\operatorname{index}(\mathcal{E})}$ for $D(\mathcal{E})$ though we are not able to define the latter object. In particular, the local index form also determines candidates for the Chern-Weil representatives $c_{k}\left(\nabla^{\operatorname{index}(\mathcal{E})}\right)$ of the Chern classes $c_{k}(\operatorname{index}(\mathcal{E}))$. Unfortunately we are not able to define natural lifts $\widehat{c}_{k}(\mathcal{E}) \in H_{\text {Del }}^{k}(B)$ of $c_{k}(\operatorname{index}(\mathcal{E}))$ with curvature $\omega\left(\hat{c}_{k}(\mathcal{E})\right)=c_{k}\left(\nabla^{\operatorname{index}(\mathcal{E})}\right)$. 
2.1.14 Assume that index $(\mathcal{E}) \in K_{k}^{*}(B)$ and $k=2 m$ or $k=2 m-1$. By Equation (1) we have

$$
c_{k}(\operatorname{index}(\mathcal{E}))_{\mathbb{R}}=\left[(-1)^{m-1}(m-1) ! \Omega^{k}(\mathcal{E})\right] .
$$

In [6, Definition 8.19] we have constructed a natural class

$$
\widehat{c}_{k}(\mathcal{E}) \in H_{\mathrm{Del}}^{k}(B)
$$

with curvature $\omega\left(\hat{c}_{k}(\mathcal{E})\right)=\Omega^{k}(\mathcal{E})$ and $v\left(\hat{c}_{k}(\mathcal{E})\right)=c_{k}(\operatorname{index}(\mathcal{E}))$. Instead of repeating the rather indirect construction [6] we give here a direct description which could be taken as definition of $\widehat{c}_{k}(\mathcal{E})$ as well. Note that $\widehat{c}_{k}(\mathcal{E})=0$ by definition if $n \not \equiv k(2)$ (recall that $n$ is the dimension of the fiber of $\mathcal{E}$ ).

2.1.15 Let $Z \in Z^{k-1}$ be a smooth cycle. We must prescribe $\widehat{c}_{k}(\mathcal{E})(Z)$. We can find a smooth manifold $X$ (not necessarily closed) of the homotopy type of a $(k-1)-$ dimensional CW-complex, a map $f: X \rightarrow B$, and a smooth $(k-1)$-cycle $Z^{\prime}$ in $X$, such that $f_{*} Z^{\prime}=Z$. We could eg take for $f: X \rightarrow B$ the inclusion of a thickening of the trace $|Z| \subset B$ of $Z$ and $Z=Z^{\prime}$. Note that $0=f^{*} \operatorname{index}(\mathcal{E})=\operatorname{index}\left(f^{*} \mathcal{E}\right)$. Therefore we can find a perturbation of the family of Dirac operators $D\left(f^{*} \mathcal{E}\right)$ by a family of selfadjoint smoothing operators $Q$ (which are odd in the even-dimensional case) such that the family $D\left(f^{*} \mathcal{E}\right)+Q$ is invertible. In [6] the pair $\left(f^{*} \mathcal{E}, Q\right)$ was called a tamed geometric family and denoted by $f^{*} \mathcal{E}_{t}$.

2.1.16 If $\mathcal{F}_{t}$ is a tamed geometric family over some base $B$, then the super connection formalism provides an eta-form $\eta\left(\mathcal{F}_{t}\right) \in \mathcal{A}(B)$ such that $d \eta\left(\mathcal{F}_{t}\right)=\Omega(\mathcal{E})$. We refer to [6] and [4] for details. The form $\eta\left(\mathcal{F}_{t}\right)$ depends on the taming. Assume that $\mathcal{F}_{t}^{\prime}$ is a second taming of the same underlying geometric family. Then the difference $\eta\left(\mathcal{F}_{t}\right)-\eta\left(\mathcal{F}_{t}^{\prime}\right)$ is a closed form. As a consequence of the index theorem for boundary tamed families [6] we know that

$$
\left[\eta\left(\mathcal{F}_{t}\right)-\eta\left(\mathcal{F}_{t}^{\prime}\right)\right]=\mathbf{c h}(x)_{\mathbb{R}}
$$

for some $x \in K^{*}(B)$. In fact, we can take $x=\operatorname{index}\left((\mathcal{F} \times I)_{\mathrm{bt}}\right)$, where the boundary taming is induced by $\mathcal{F}_{t}$ and $\mathcal{F}_{t}^{\prime}$.

2.1.17 We can now prescribe $\widehat{c}_{k}(\mathcal{E})(Z)$ as follows.

\section{Definition 2.2}

$$
\widehat{c}_{k}(\mathcal{E})(Z):=\left[(-1)^{m-1}(m-1) ! \int_{Z^{\prime}} \eta^{k-1}\left(f^{*} \mathcal{E}_{t}\right)\right] \in \mathbb{R} / \mathbb{Z} .
$$

In order to see that $\widehat{c}_{k}(\mathcal{E})$ is well defined note that $(m-1) ! \mathbf{c h}_{k}$ is an integral cohomology class. Therefore the right-hand side does not depend on the choice of the taming. One 
also checks independence of $f, X, Z^{\prime}$. The relation $\omega\left(\widehat{c}_{k}(\mathcal{E})\right)=\Omega^{k}(\mathcal{E})$ follows from $d \eta\left(f^{*} \mathcal{E}_{t}\right)=f^{*} \Omega(\mathcal{E})$.

2.1.18 Up to this point we have not employed the fact that the geometric bundle $\mathbf{W}=\left(W, \nabla^{W}, h^{W}\right)$ comes with a real structure $Q$. Because of the existence of $Q$ the geometric bundle $\mathbf{W}$ is isomorphic to its hermitian conjugate $\overline{\mathbf{W}}$. We conclude from the general equality

$$
\operatorname{ch}_{2 k}\left(\nabla^{\bar{V}}\right)=(-1)^{k} \mathbf{c h}_{2 k}\left(\nabla^{V}\right)
$$

that $\operatorname{ch}_{l}\left(\nabla^{W}\right)=0$ if $l \not \equiv 0(4)$.

2.1.19 Recall that $n=\operatorname{dim}(E)-\operatorname{dim}(B)$.

Lemma 2.3 If $k+n \not \equiv 0(4)$, then $\Omega^{k}(\mathcal{E})=0$.

Proof We have

$$
\Omega(\mathcal{E})=\int_{E / B} \widehat{A}\left(\nabla^{T^{v} \pi}\right) \mathbf{c h}\left(\nabla^{W}\right) .
$$

Since the form $\hat{A}\left(\nabla^{T^{v}} \pi\right)$ is nontrivial only in degrees $4 l, l \geq 0$, we immediately see that $\Omega^{k}(\mathcal{E})=0$ if $k+n \not \equiv 0(4)$.

2.1.20 We call a class $\hat{x} \in H_{\text {Del }}^{k}(B)$ flat if $\omega(\hat{x})=0$. By Lemma 2.3 the class $\widehat{c}_{k}(\mathcal{E})$ is flat if $k+n \equiv 2(4)$. The Deligne cohomology of $B$ fits into the exact sequence (see Cheeger-Simons [9])

$$
0 \rightarrow H^{k-1}(B, \mathbb{R} / \mathbb{Z}) \stackrel{\hat{b}}{\rightarrow} H_{\mathrm{Del}}^{k}(B) \stackrel{\omega}{\rightarrow} \mathcal{A}^{k}(B)
$$

such that $v \circ \hat{b}: H^{k-1}(B, \mathbb{R} / \mathbb{Z}) \rightarrow H^{k}(B, \mathbb{Z})$ is the Bockstein operator associated to the exact sequence of coefficients

$$
0 \rightarrow \mathbb{Z} \rightarrow \mathbb{R} \rightarrow \mathbb{R} / \mathbb{Z} \rightarrow 0
$$

By (3), a flat class in $H_{\text {Del }}^{k}(B)$ can be considered as a class in $H^{k-1}(B, \mathbb{R} / \mathbb{Z})$. From now on we consider $H^{k-1}(B, \mathbb{R} / \mathbb{Z})$ as a subset of $H_{\text {Del }}^{k}(B)$ and do not write $\hat{b}$ anymore.

2.1.21 The first assertion of the following proposition is just the conclusion of the preceding discussion.

Proposition 2.4 (1) Let $\mathcal{E}$ be a geometric family over $B$ such that the geometric twisting bundle $\mathbf{W}$ admits a real structure. Let $k \geq 0$ and assume that index $(\mathcal{E}) \in$ $K_{k}^{*}(B)$. If furthermore $k+n \equiv 2(4)$ (where $n=\operatorname{dim}(E)-\operatorname{dim}(B)$ is the fiber dimension of $\mathcal{E}$ ) then $\widehat{c}_{k}(\mathcal{E}) \in H_{\text {Del }}^{k}$ is flat and therefore gives rise to a class in $H^{k-1}(B, \mathbb{R} / \mathbb{Z})$. 
(2) The class $\widehat{c}_{k}(\mathcal{E}) \in H^{k-1}(B, \mathbb{R} / \mathbb{Z})$ is independent of the geometric structures, ie it only depends on the differentiable fiber bundle $E \rightarrow B$, the choice of spin structure and orientation of the vertical bundle $T^{v} \pi$, and on the real vector bundle $W_{R}$.

Proof In order to show the independence of the geometric structures we argue using the connectedness of the space $P$ of these structures. We can set up a universal family $\mathcal{E}_{\text {univ }}$ over $P \times B$ and define $\widehat{c}_{k}\left(\mathcal{E}_{\text {univ }}\right) \in H^{k-1}(P \times B, \mathbb{R} / \mathbb{Z})$. It follows from the homotopy invariance of the cohomology functor and the naturality of the construction of these classes with respect to pullback, that the specializations of $\widehat{c}_{k}\left(\mathcal{E}_{\text {univ }}\right)$ at different parameter points $p, q$ are cohomologous on the one hand, and give the classes $\widehat{c}_{k}\left(\mathcal{E}_{p}\right)$ and $\widehat{c}_{k}\left(\mathcal{E}_{q}\right)$ associated to the families $\mathcal{E}_{p}$ and $\mathcal{E}_{q}$ equipped with geometric structures given by $p$ and $q$, respectively, on the other hand.

2.1.22 The main goal of the present paper is to understand the nature of the class $\widehat{c}_{k}(\mathcal{E}) \in H^{k-1}(B, \mathbb{R} / \mathbb{Z})$ in terms of the topology of the geometric family.

\subsection{The real index}

2.2.1 The group $K O^{0}(B)$ is defined as the group completion of the semigroup of isomorphism classes of real vector bundles over $B$. The functor $B \mapsto K O^{0}(B)$ extends to an 8-periodic multiplicative cohomology theory $K O^{*}$. Complexification of real vector bundles induces a natural transformation $c_{B}: K O^{0}(B) \rightarrow K^{0}(B)$ which extends to a natural transformation $c_{B}: K O^{*}(B) \rightarrow K O^{*}(B)$ of multiplicative cohomology theories.

If $k+n \not \equiv 0(4)$, then the composition

$$
K O^{-n}(B) \stackrel{c_{B}}{\longrightarrow} K^{-n}(B) \stackrel{\mathbf{c h}_{k}}{\longrightarrow} H^{k}(B, \mathbb{Q})
$$

vanishes.

2.2.2 In view of this observation the desirable explanation of the fact that $\Omega^{k}(\mathcal{E})=0$ if $k+n \equiv 2(4)$ is that $\operatorname{index}(\mathcal{E}) \in K^{-n}(B)$ is in fact of the form $c_{B}\left(\operatorname{index}_{\mathbb{R}}(\mathcal{E})\right)$ for a real refinement of the index index $\mathbb{R}_{\mathbb{R}}(\mathcal{E}) \in K O^{-n}(B)$. In fact, the spinor bundle carries additional structures which are "preserved" upon twisting by real bundles. Using these structures we can indeed refine the index $\operatorname{index}(\mathcal{E}) \in K^{-n}(B)$ to a class $\operatorname{index}_{\mathbb{R}}(\mathcal{E}) \in K O^{-n}(B)$.

For the purpose of illustration we sketch the construction of $\operatorname{index}_{\mathbb{R}}(\mathcal{E})$. Although this is well known, the following exposition is designed to be a useful reference for the interested reader. 
2.2.3 Depending on the class of $n$ modulo 8 we are going to use quite different pictures of $K O^{-n}(B)$. We make use of the real Clifford algebras $C^{p, q}$ associated to $\mathbb{R}^{p+q}$ with quadratic form $-x_{1}^{2}-\cdots-x_{p}^{2}+x_{p+1}^{2}+\cdots+x_{p+q}^{2}$.

In one picture an element of $K O^{n}$ is represented as a family of selfadjoint odd Fredholm operators on a graded $C^{n, 0}$-module. Another representation is as a family of antisymmetric Fredholm operators which anticommute with an action of $C^{0, n-1}$. In this case there is no grading. Finally an element of $K^{1}(B)$ is represented by a family of selfadjoint Fredholm operators (and there is again no grading). We refer to Atiyah-Singer [3] and Karoubi [11] for further details.

2.2.4 $n \equiv 0(8)$

The spinor bundle $\mathcal{S}\left(T^{v} \pi\right)$ is the complexification of a real spinor bundle $\mathcal{S}_{\mathbb{R}}\left(T^{v} \pi\right)$. Thus $\mathcal{V}=\mathcal{S}\left(T^{v} \pi\right) \otimes W$ is the complexification of $\mathcal{V}_{\mathbb{R}}:=\mathcal{S}_{\mathbb{R}}\left(T^{v} \pi\right) \otimes W_{\mathbb{R}}$. The Dirac operator $D(\mathcal{E})$ comes from the Dirac operator $D_{\mathbb{R}}(\mathcal{E})$ on $\mathcal{V}_{\mathbb{R}}$. The refined index index $\mathbb{R}_{\mathbb{R}}(\mathcal{E}) \in K O^{0}(B)$ is just the index of the family of real Fredholm operators $D_{\mathbb{R}}(\mathcal{E})^{+}$.

2.2.5 $n \equiv 1(8)$

The spinor bundle $\mathcal{S}\left(T^{v} \pi\right)$ admits a real structure, which anticommutes with Clifford multiplication. It induces a real structure on $\mathcal{V}$ which anticommutes with $D(\mathcal{E})$. Let $\mathcal{V}_{\mathbb{R}}$ be again the real +1 -eigenbundle of the real structure on $\mathcal{V}$. The operator $i D(\mathcal{E})$ commutes with this real structure and therefore induces an antisymmetric operator $D(\mathcal{E})_{\mathbb{R}}$ on $\mathcal{V}_{\mathbb{R}}$. This family represents index $\mathbb{R}(\mathcal{E}) \in K O^{-1}(B)$.

2.2.6 $n \equiv 2(8)$

The spinor bundle $\mathcal{S}\left(T^{v} \pi\right)$ has a quaternionic structure which is odd with respect to the $\mathbb{Z} / 2 \mathbb{Z}$-grading and commutes with Clifford multiplication. Thus we obtain an induced quaternionic structure $J$ on $\mathcal{V}$. We consider $D(\mathcal{E})_{\mathbb{R}}:=J D(\mathcal{E})$ as an antisymmetric real operator on $\mathcal{V}_{\mathbb{R}}^{+}$. It anticommutes with the action of $C^{0,1}$ which is induced by multiplication by $i$. Therefore the family $D(\mathcal{E})_{\mathbb{R}}$ together with the $C^{0,1}$-module structure represents index $\mathbb{R}_{\mathbb{R}}(\mathcal{E}) \in K O^{-2}(B)$.

2.2.7 $n \equiv 3(8)$

The spinor bundle carries a quaternionic structure which commutes with Clifford multiplication. We get an induced quaternionic structure $J$ on $\mathcal{V}$ commuting with $D(\mathcal{E})$. The antisymmetric operator $D(\mathcal{E})_{\mathbb{R}}:=i D(\mathcal{E})$ anticommutes with the action of $C^{0,2}$ generated by $J$ and $i J$. Therefore the family $D(\mathcal{E})_{\mathbb{R}}$ on $\mathcal{V}_{\mathbb{R}}$ together with the $C^{0,2}$-module structure represents index $\mathbb{R}_{\mathbb{R}}(\mathcal{E}) \in \mathrm{KO}^{-3}(B)$. 
2.2.8 $n \equiv 4(8)$

The spinor bundle $\mathcal{S}\left(T^{v} \pi\right)$ carries a quaternionic structure which commutes with the grading and Clifford multiplication. It induces a quaternionic structure $J$ on $\mathcal{V}$ which commutes with $D(\mathcal{E})$. We consider the antisymmetric operator $D(\mathcal{E})_{\mathbb{R}}:=i D(\mathcal{E})$ on the bundle $\mathcal{V}_{\mathbb{R}}$ which anticommutes with the Clifford algebra $C^{0,3}$ generated by $i, J, i J$. Therefore the family $D(\mathcal{E})_{\mathbb{R}}$ on $\mathcal{V}_{\mathbb{R}}$ together with the $C^{0,3}$-module structure represents $\operatorname{index}_{\mathbb{R}}(\mathcal{E}) \in K O^{-4}(B)$.

2.2.9 $n \equiv 5(8)$

The spinor bundle $\mathcal{S}\left(T^{v} \pi\right)$ carries a quaternionic structure which anticommutes with the Clifford multiplication. It induces a quaternionic structure $J$ on $\mathcal{V}$ which anticommutes with $D(\mathcal{E})$. We form the real selfadjoint operator

$$
D(\mathcal{E})_{\mathbb{R}}:=\left(\begin{array}{cc}
D(\mathcal{E}) & 0 \\
0 & -D(\mathcal{E})
\end{array}\right)
$$

on $\mathcal{V}_{\mathbb{R}} \oplus \mathcal{V}_{\mathbb{R}}$ with its standard odd grading

$$
\left(\begin{array}{ll}
0 & 1 \\
1 & 0
\end{array}\right)
$$

This operator commutes with the Clifford algebra $C^{3,0}$ generated by

$$
\left(\begin{array}{cc}
0 & J \\
-J & 0
\end{array}\right),\left(\begin{array}{cc}
0 & i J \\
-i J & 0
\end{array}\right),\left(\begin{array}{cc}
1 & 0 \\
0 & -1
\end{array}\right)
$$

The $C^{3,0}$-equivariant operator $D(\mathcal{E})_{\mathbb{R}}$ represents index $\mathbb{R}_{\mathbb{R}}(\mathcal{E}) \in K O^{3}(B) \cong K O^{-5}(B)$.

2.2.10 $n \equiv 6(8)$

The spinor bundle $\mathcal{S}\left(T^{v} \pi\right)$ carries a real structure which anticommutes with the grading. In induces a real structure $Q$ on $\mathcal{V}$ which is odd and commutes with $D(\mathcal{E})$. We consider the selfadjoint operator $D(\mathcal{E})_{\mathbb{R}}:=D(\mathcal{E})$ on $\mathcal{V}_{\mathbb{R}}$. This bundle is $\mathbb{Z} / 2 \mathbb{Z}$-graded and admits an action of $C^{2,0}$ generated by $Q$ and $i Q$ commuting with $D(\mathcal{E})_{\mathbb{R}}$. The $C^{2,0}$-equivariant operator $D(\mathcal{E})_{\mathbb{R}}$ represents index $(\mathcal{E})_{\mathbb{R}} \in K O^{2}(B) \cong K O^{-6}(B)$.

2.2.11 $n \equiv 7(8)$

The spinor bundle $\mathcal{S}\left(T^{v} \pi\right)$ admits a real structure which commutes with the Clifford multiplication. In induces a real structure $Q$ on $\mathcal{V}$ which commutes with $D(\mathcal{E})$. We consider the real symmetric operator $D(\mathcal{E})_{\mathbb{R}}$ which is obtained by restricting $D(\mathcal{E})$ to the 1-eigenbundle of $Q$. The operator $D(\mathcal{E})_{\mathbb{R}}$ represents $\operatorname{index}_{\mathbb{R}}(\mathcal{E}) \in K O^{1}(B) \cong$ $\mathrm{KO}^{-7}(B)$. 


\section{The analytic invariant}

\subsection{Construction of a natural transformation $d_{B, k-1}^{n}$}

3.1.1 Recall that complexification of real vector bundles induces a natural transformation of multiplicative cohomology theories $c_{B}: K O^{n}(B) \rightarrow K^{n}(B)$. The real index $\operatorname{index}_{\mathbb{R}}(\mathcal{E}) \in K O^{-n}(B)$ is a refinement of $\operatorname{index}(\mathcal{E}) \in K^{-n}(B)$ in the sense that

$$
c_{B}\left(\operatorname{index} \mathbb{R}_{\mathbb{E}}(\mathcal{E})\right)=\operatorname{index}(\mathcal{E}) .
$$

3.1.2 For $k \geq 0$ and $n \in \mathbb{Z}$ we define the group $U_{k}^{n}(B)$ by the following exact sequence

$$
0 \rightarrow U_{k}^{n}(B) \rightarrow K O^{n}(B) \stackrel{q_{B}}{\rightarrow} K^{n}(B) / K_{k}^{n}(B),
$$

where $q_{B}$ is the composition of $c_{B}$ with the projection onto the quotient. We also define $U_{\infty}^{n}(B)$ by the exact sequence

$$
0 \rightarrow U_{\infty}^{n}(B) \rightarrow K O^{n}(B) \rightarrow K^{n}(B) .
$$

Since $q_{B}$ is a natural transformation the association $B \mapsto U_{k}^{n}(B)$ extends to a functor with values in abelian groups.

3.1.3 Assume that $k-n \equiv 2(4)$.

Definition 3.1 We define the natural transformation

$$
d_{B, k-1}^{n}: U_{k}^{n}(B) \rightarrow H^{k-1}(B, \mathbb{R} / \mathbb{Z})
$$

by the requirement that

$$
d_{B, k-1}^{n}(x):=\widehat{c}_{k-1}(\mathcal{E}) \text {, }
$$

where $\mathcal{E}$ is any geometric family of dimension $8 l-n, l \in \mathbb{Z}$, such that $x=\operatorname{index}_{\mathbb{R}}(\mathcal{E})$.

3.1.4 We must check that the definition of $d_{B, k-1}^{n}$ makes sense.

Proposition 3.2 If $k-n \equiv 2(4)$, then there is a unique homomorphism

$$
d_{B, k-1}^{n}: U_{k}^{n}(B) \rightarrow H^{k-1}(B, \mathbb{R} / \mathbb{Z})
$$

such that $d_{B, k-1}^{n}\left(\operatorname{index}_{\mathbb{R}}(\mathcal{E})\right)=\widehat{c}_{k}(\mathcal{E})$ for any geometric family over $B$ of dimension $8 l-n, l \in \mathbb{Z}$, with index $(\mathcal{E}) \in U_{k}^{n}(B)$. This homomorphism is natural with respect to continuous maps. 
Proof The essential parts of the proof are given in Lemma 3.3 and Lemma 3.4 below. It immediately follows from these Lemmas that for given $B$ there exist a unique map $d_{B, k-1}^{n}$ satisfying the requirement. Additivity and naturality with respect to smooth maps of $d_{B, k-1}^{n}$ follows from naturality and additivity of the class $\widehat{c}_{k}(\mathcal{E})$. But then naturality extends to continuous maps since $U_{k}^{n}(\cdot)$ as well as $H^{k-1}(\cdot, \mathbb{R} / \mathbb{Z})$ are weak homotopy functors.

3.1.5 The following lemma establishes that $d_{B, k}^{n}$ is well defined.

Lemma 3.3 If $\operatorname{index}_{\mathbb{R}}(\mathcal{E})=0$, then $\widehat{c}_{k}(\mathcal{E})=0$.

Proof Assume that index $\mathbb{R}(\mathcal{E})=0$. In this case we can find a smoothing perturbation of the real operator $D(\mathcal{E})_{\mathbb{R}}$ which is invertible. We call this perturbation a real taming. By complexification a real taming induces a taming $\mathcal{E}_{t}$ which is compatible with the additional symmetries determining the real structure.

These additional symmetries imply that the Chern form of the Bismut super connection associated to $D(\mathcal{E})$ and its tamed perturbation vanishes. Since the $\eta$-form is derived from this Chern form we conclude that $\eta\left(\mathcal{E}_{t}\right)=0$ if the taming is induced from a real taming. The assertion of the Lemma now follows from the description of $\widehat{c}_{k}(\mathcal{E})$ in terms of the $\eta$-form (see Section 2.1.17).

3.1.6 The following lemma shows how to realize $K$-theory classes.

Lemma 3.4 If $x \in K O^{n}(B)$, then there exists a geometric family $\mathcal{E}$ as above such that $\operatorname{index}_{\mathbb{R}}(\mathcal{E})=x$.

Proof By the periodicity of $K O^{*}(B)$ we can assume that $n<0$. By definition,

$$
K O^{n}(B)=\widetilde{K O^{n}}\left(B_{+}\right)=\widetilde{K O^{0}}\left(\Sigma^{n} B_{+}\right),
$$

where $B_{+}$is obtained from $B$ by adjoining an additional base point, and $\widetilde{K O}^{n}\left(B_{+}\right)$ denotes the reduced $K O$-theory.

Let now $x \in K O^{n}(B)$ correspond to $\tilde{x} \in \widetilde{K O^{0}}\left(\Sigma^{n} B_{+}\right)$. Let $p: S^{n} \times B_{+} \rightarrow \Sigma^{n} B_{+}$be the natural projection and $W_{\mathbb{R}}=W_{\mathbb{R}}^{+} \oplus W_{\mathbb{R}}^{-}$be the real $\mathbb{Z} / 2 \mathbb{Z}$-graded vector bundle over $S^{n} \times B_{+}$representing $p^{*} \tilde{x}$.

We form two geometric families $\mathcal{E}^{ \pm}$with underlying bundle $S^{n} \times B_{+} \rightarrow B_{+}$with its standard fibrewise orientation and spin structure and with the real twisting bundle $W_{\mathbb{R}}^{ \pm}$. Then by Bott periodicity or the index theorem, $x=\operatorname{index}_{\mathbb{R}}\left(\mathcal{E}^{+} \cup_{B_{+}} \mathcal{E}^{-}\right)_{\left.\right|_{B}}$. 


\subsection{Some properties of $d_{B, k-1}^{n}$}

3.2.1 We approach the study of the natural transformation $d_{B, k-1}^{n}$ from two sides. First, in view of its definition through the analysis of families of Dirac operators we use mainly analytical arguments in order to show some simple properties of this transformation. This is the subject to the present section.

A finer study in Section 4 leading to a complete understanding of the transformation uses methods from topology and the observation that a natural transformation comes from a universal one between suitable classifying spaces. It should be noted that most results of this section, the important exception being Corollary 3.7, will also follow from the topological description, and will not be needed to derive this description.

3.2.2 There is a natural transformation

$$
r_{B}: K^{*}(B) \rightarrow K O^{*}(B) .
$$

It is determined by the special case $r_{B}: K^{0}(B) \rightarrow K O^{0}(B)$ which associates to a class represented by a complex vector bundle the class represented by the underlying real vector bundle. It is easy to see that

$$
r_{B} \circ c_{B}=2
$$

(multiplication by 2).

3.2.3 We now prove the following proposition.

Proposition 3.5 We have $2 d_{B, k-1}^{n}=0$.

Proof Fix $x \in U_{k}^{n}(B)$. The homology class $d_{B, k-1}^{n}(x) \in H^{k-1}(B, \mathbb{R} / \mathbb{Z})$ is determined by its values on all smooth cycles $Z \in Z^{k-1}$ on $B$.

Given a $(k-1)$-cycle $Z$ there exists a manifold $X$ which is homotopy equivalent to a $(k-1)$-dimensional CW-complex, a smooth map $f: X \rightarrow B$, and a $(k-1)$-cycle $Z^{\prime}$ in $X$ such that $f_{*} Z^{\prime}=Z$ (compare 2.1.15). By the naturality of $d_{\cdot, k-1}^{n}$ we have

$$
2 d_{B, k-1}^{n}(x)(Z)=2 f^{*} d_{B, k-1}^{n}(x)\left(Z^{\prime}\right)=d_{X, k-1}^{n}\left(2 f^{*} x\right)\left(Z^{\prime}\right) .
$$

It thus suffices to show that $2 f^{*} x=0$.

Since $f^{*} x \in U_{k}^{n}(X)$ and $X$ is (up to homotopy equivalence) $(k-1)$-dimensional we have $c_{X}\left(f^{*} x\right)=0$. This implies $0=r_{X} \circ c_{X}\left(f^{*} x\right)=2 f^{*} x$.

3.2.4 We have defined $d_{B, k-1}^{n}$ in terms of eta forms of families of Dirac operators. In the following we show that it suffices to understand eta forms for zero-dimensional families. Note that the latter are essentially objects of linear algebra. 
3.2.5 If $x \in U_{\infty}^{-n}(B)$, then there exist a real $\mathbb{Z} / 2 \mathbb{Z}$-graded vector bundle over $\Sigma^{n} B_{+}$ which represents the class $\tilde{x} \in \widetilde{K O}^{0}\left(\Sigma^{n} B_{+}\right)$corresponding to $x$ under the identification $K O^{-n}(B) \cong \widetilde{K O^{0}}\left(\Sigma^{n} B_{+}\right)$. Let $W_{\mathbb{R}}=W_{\mathbb{R}}^{+} \oplus W_{\mathbb{R}}^{-}$be the pullback of this bundle to $S^{n} \times B$ under the natural map $S^{n} \times B \rightarrow \Sigma^{n} B_{+}$.

The bundle $\pi: S^{n} \times B \rightarrow B$ has a natural fibrewise orientation and spin structure. The round metric of $S^{n}$ induces a fibrewise metric $g^{T^{v}} \pi$. The canonical decomposition $T\left(S^{n} \times B\right)=\operatorname{pr}_{S^{n}}^{*} T S^{n} \oplus \operatorname{pr}_{B}^{*} T B$ yields the horizontal distribution $\operatorname{pr}_{B}^{*} T B$. After choosing geometric bundles $\mathbf{W}_{\mathbb{R}}^{ \pm}=\left(W_{\mathbb{R}}^{ \pm}, \nabla_{\mathbb{R}}^{ \pm}, h^{W_{\mathbb{R}}^{ \pm}}\right)$we obtain the geometric family $\mathcal{E}^{ \pm}$and $\mathcal{E}:=\mathcal{E}^{+} \cup_{B}\left(\mathcal{E}^{-}\right)^{\text {op }}$ over $B$, with underlying bundle $S^{n} \times B$, such that $\operatorname{index}_{\mathbb{R}}(\mathcal{E})=x$.

3.2.6 Since $c_{B}(x)=0$ by assumption we know that the complexification $W$ of $W_{\mathbb{R}}$ represents the trivial element $0=[W] \in K^{0}\left(S^{n} \times B\right)$. Thus (possibly after adding a trivial bundle of formal dimension zero) the bundle $\mathbf{W}$ admits an odd unitary selfadjoint (not necessary parallel) automorphism $U$.

3.2.7 The bundles $\mathbf{W}_{\mathbb{R}}^{ \pm}$give rise to geometric families $\mathcal{F}^{ \pm}$over $S^{n} \times B$ where the underlying zero-dimensional fiber bundle is id: $S^{n} \times B \rightarrow S^{n} \times B$, and the twisting bundle is $\mathbf{W}_{\mathbb{R}}^{ \pm}$. We let $\mathcal{F}:=\mathcal{F}^{+} \cup_{S^{n} \times B}\left(\mathcal{F}^{-}\right)^{\text {op }}$. Then we have $\operatorname{index}_{\mathbb{R}}(\mathcal{F})=\left[W_{\mathbb{R}}\right] \in$ $K O^{0}\left(S^{n} \times B\right)$ and index $(\mathcal{F})=[W]=0 \in K^{0}\left(S^{n} \times B\right)$. The automorphism $U$ gives a taming $\mathcal{F}_{t}$ of $\mathcal{F}$. In particular, we have a well-defined form $\eta\left(\mathcal{F}_{t}\right) \in \mathcal{A}\left(S^{n} \times B\right)$ such that $\operatorname{d\eta }\left(\mathcal{F}_{t}\right)=\Omega(\mathcal{F})=\mathbf{c h}\left(\nabla^{W}\right)$.

3.2.8 For $r>0$ the operator $r U$ can be considered as a sort of taming of the family $\mathcal{E}$. It is not a taming in the strong sense since $U$ is not smoothing along the fibers of $\pi$. Rather it is a local taming in the sense of [6]. Local index theory works for local tamings as well. We let $\mathcal{E}_{t}(r)$ be the geometric family $\mathcal{E}$ tamed with $r U$ and let $\eta\left(\mathcal{E}_{t}(r)\right)$ be the associated $\eta$-form.

3.2.9 We now establish a proposition.

Proposition 3.6 We have

$$
\lim _{r \rightarrow \infty} \eta\left(\mathcal{E}_{t}(r)\right)=\int_{S^{n} \times B / B} \eta(\mathcal{F}) .
$$

Proof This assertion is proved using the adiabatic limit techniques developed eg in [7]. The general method gives

$$
\lim _{r \rightarrow \infty} \eta\left(\mathcal{E}_{t}(r)\right)=\int_{S^{n} \times B / B} \hat{A}\left(\nabla^{T^{v} \pi}\right) \eta\left(\mathcal{F}_{t}\right)
$$

The result now follows from $\hat{A}\left(\nabla^{T^{v} \pi}\right)=\operatorname{pr}_{S^{n}}^{*} \hat{A}\left(\nabla^{T S^{n}}\right)=1$, since $\hat{A}\left(\nabla^{T S^{n}}\right)=1$ for the round metric (which is also locally conformally flat). 
3.2.10 A closed form $\omega \in \mathcal{A}^{k-1}(B)$ represents a class $[\omega] \in H^{k-1}(B, \mathbb{R})$. By $[\omega]_{\mathbb{R} / \mathbb{Z}} \in H^{k-1}(B, \mathbb{R} / \mathbb{Z})$ we denote its natural image. Let $m$ be determined by $2 m=k$ or $2 m=k+1$. Then definition (2) together with Proposition 3.6 implies the following corollary.

Corollary 3.7 With the notation above

$$
d_{B, k-1}^{-n}(x)=\left[(-1)^{m-1}(m-1) ! \int_{S^{n} \times B / B} \eta^{k+n-1}(\mathcal{F})\right]_{\mathbb{R} / \mathbb{Z}} .
$$

In fact, in view of Proposition 3.5 we could also omit the sign $(-1)^{m-1}$.

3.2.11 We consider the sequence

$$
0 \rightarrow \mathbb{Z} \rightarrow \mathbb{R} \rightarrow \mathbb{R} / \mathbb{Z} \rightarrow 0
$$

and let $\beta^{\prime \prime}: H^{k-1}(B, \mathbb{R} / \mathbb{Z}) \rightarrow H^{k}(B, \mathbb{Z})$ be the associated Bockstein operator. Recall from 2.1.20, that $\beta^{\prime \prime}$ coincides with the composition

$$
H^{k-1}(B, \mathbb{R} / \mathbb{Z}) \rightarrow H_{\text {Del }}^{k}(B) \stackrel{v}{\rightarrow} H^{k}(B, \mathbb{Z}) .
$$

Proposition 3.8 For $x \in U_{4 k+2}^{0}(B)$ we have

$$
\left(\beta^{\prime \prime} \circ d_{4 k+1}^{0}\right)(x)=\left(c_{4 k+2} \circ c_{B}\right)(x) .
$$

Proof We write $x=\operatorname{index}_{\mathbb{R}}(\mathcal{E})$ for a suitable geometric family $\mathcal{E}$ over $B$. Then we have the chain of equalities

$$
\left(\beta^{\prime \prime} \circ d_{4 k+1}^{0}\right)(x)=\left(v \circ \widehat{c}_{4 k+2}\right)(\mathcal{E})=c_{4 k+2}(\operatorname{index}(\mathcal{E}))=\left(c_{4 k+2} \circ c_{B}\right)(x) .
$$

\subsection{Nontrivial examples}

In Section 4.4 we will give a complete description of the universal classes $d_{\cdot, k}^{n}$, which also decides when exactly these classes can be nontrivial. In this section, we want to construct explicit and easy nontrivial examples over low dimensional spheres as base manifolds.

Algebraic 83 Geometric Topology, Volume 8 (2008) 
3.3.1 Let MSpin* be the spin bordism cohomology theory and $\alpha$ : MSpin* $\rightarrow K O^{*}$ be the $\alpha$-genus introduced by Hitchin [10]. Note that $\alpha: \operatorname{MSpin}^{*}(*) \rightarrow K O^{*}(*)$ is surjective. If $E$ is a closed spin manifold, then we write $\alpha(E)$ for the result of $\alpha$ applied to the class $[E] \in \operatorname{MSpin}^{*}(*)$ represented by $E$.

The most important common feature of the following examples is that they all come with a trivial twisting bundle. In other words, the respective geometric family $\mathcal{E}$ represents an element $[E, \pi] \in \operatorname{MSpin}^{-n}\left(S^{i}\right)$ (with $n \in \mathbb{N}_{0}$ and $i \in\{0,1,2\}$ depending on the case), such that index $\mathbb{R}_{\mathbb{R}}(\mathcal{E})=\alpha([E, \pi])$.

3.3.2 $n \equiv 1(8), k=1$

Let $E$ be a closed spin manifold of dimension $n \equiv 1(8)$ with $\alpha(E)=1 \in K O^{-1}(*) \cong$ $\mathbb{Z} / 2 \mathbb{Z}$. Such manifolds exist by the 3.3.1.

We choose a Riemannian metric $g^{T E}$. Then we consider $E$ as a geometric family $\mathcal{E}$ over the point $*$ with the trivial twisting bundle $W_{\mathbb{R}}=E \times \mathbb{R}$. We claim that $\widehat{c}_{1}(\mathcal{E}) \neq 0$.

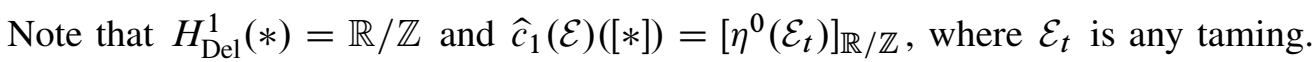
The degree 0 part $\eta^{0}\left(\mathcal{E}_{t}\right)$ is defined even for a pretamed manifold in the sense of [6], and if the pretaming is trivial, then it is one half of the usual Atiyah-Patodi-Singer eta invariant [2]. Now we have $\eta^{0}(\mathcal{E})=0$ since $D(\mathcal{E})$ anticommutes with the real structure and thus has symmetric spectrum. Since by spectral flow (taming essentially means that the underlying operators are invertible)

we see that

$$
\begin{aligned}
{\left[\eta^{0}\left(\mathcal{E}_{t}\right)-\eta^{0}(\mathcal{E})\right]_{\mathbb{R} / \mathbb{Z}} } & =\left[\frac{1}{2} \operatorname{dim} \operatorname{ker} D(\mathcal{E})\right]_{\mathbb{R} / \mathbb{Z}} \\
{\left[\eta^{0}\left(\mathcal{E}_{t}\right)\right]_{\mathbb{R} / \mathbb{Z}} } & =\left[\frac{1}{2} \operatorname{dim} \operatorname{ker} D(\mathcal{E})\right]_{\mathbb{R} / \mathbb{Z}} .
\end{aligned}
$$

The condition $\alpha(E) \neq 0$ says that $\operatorname{dim} \operatorname{ker} D(\mathcal{E}) \equiv 1(2)$. Therefore, $d_{*, 0}^{1}\left(\operatorname{index}_{\mathbb{R}}(\mathcal{E})\right)=$ $\left[\eta^{0}\left(\mathcal{E}_{t}\right)\right]_{\mathbb{R} / \mathbb{Z}}=\left[\frac{1}{2}\right]_{\mathbb{R} / \mathbb{Z}} \neq 0 \in H^{0}(*, \mathbb{R} / \mathbb{Z})$.

3.3.3 $n \equiv 0(8), k=2$

We consider a family $E \rightarrow S^{1}$ of closed spin manifolds with fiber dimension $n \equiv 0(8)$ and $\alpha(E) \neq 0$. Indeed for any given spin manifold $M$ of dimension $n \equiv 0(8)$ such a bundle with fiber $M$ exists by [10]. We will in addition assume that

$$
\alpha(M) \stackrel{\text { Def }}{=} \widehat{A}(M)=0 .
$$

We equip $E$ with geometric structures and consider the trivial twisting bundle $E \times W_{\mathbb{R}}$. Let $\mathcal{E}$ denote the corresponding geometric family over $S^{1}$. Since $\alpha(M)=0$ we have $\operatorname{index}(\mathcal{E}) \in K_{2}^{0}\left(S^{1}\right)=0$ and thus index $\mathbb{R}_{\mathbb{R}}(\mathcal{E}) \in U_{2}^{0}\left(S^{1}\right)$. 
We claim that $\widehat{c}_{2}(\mathcal{E}) \neq 0$. We consider a taming $\mathcal{E}_{t}$. For $a>0$ the standard metric $g^{T S^{1}}$ of $S^{1}$ and the horizontal distribution of $\mathcal{E}$ induces a Riemannian metric $g_{a}^{T E}=$

$g^{T^{v} \pi} \oplus a \pi^{*} g^{T S^{1}}$ on the total space $E$. Since $E$ is spin we can consider the total Dirac operator $D(a)$ on $E$ and its perturbation $D_{t}(a)$ which is induced by the taming. In the adiabatic limit $a \rightarrow 0$ the operator $D_{t}(a)$ becomes invertible. In other words, for small $a$ the perturbation $D_{t}(a)$ is induced by a local taming. As in [5], we have

$$
\left[\lim _{a \rightarrow 0} \eta^{0}\left(D_{t}(a)\right)\right]_{\mathbb{R} / \mathbb{Z}}=\left[\int_{S^{1}} \eta^{1}\left(\mathcal{E}_{t}\right)\right]_{\mathbb{R} / \mathbb{Z}}=d_{S^{1}, 1}^{n}\left(\operatorname{index}_{\mathbb{R}}(\mathcal{E})\right)\left(\left[S^{1}\right]\right) \in \mathbb{R} / \mathbb{Z} .
$$

For sufficiently small $a$ the class $\left[\eta^{0}\left(D_{t}\right)(a)\right]_{\mathbb{R} / \mathbb{Z}}$ is independent of the adiabatic parameter. As in 3.3.2, since $\alpha(E) \neq 0$ and $\eta^{0}(D(a))=0$ we have $\left[\eta^{0}\left(D_{t}(a)\right)\right]_{\mathbb{R} / \mathbb{Z}}=$ $\left[\frac{1}{2}\right]_{\mathbb{R} / \mathbb{Z}}$. Thus $d_{S^{1}, 1}^{n} \neq 0 \in H^{1}\left(S^{1}, \mathbb{R} / \mathbb{Z}\right)$.

This result can be interpreted as follows: The holonomy of the determinant line bundle of $\mathcal{E}$ is $-1 \in U(1)$.

3.3.4 $n=7(8), k=3$

We consider a family $E \rightarrow S^{2}$ of closed spin manifolds with fiber dimension $n \equiv 7(8)$ and $\alpha(E) \neq 0$. Such a family exists [10] for any given closed spin manifold $M$ of dimension $n \equiv 7(8)$. We choose geometric structures and consider the trivial twisting bundle $E \times \mathbb{R}$. In this way we obtain a geometric family $\mathcal{E}$. Since $K^{1}\left(S^{2}\right)=0$ we have $\operatorname{index}(\mathcal{E})=0$ and therefore $\operatorname{index}_{\mathbb{R}}(\mathcal{E}) \in U_{3}^{7}\left(S^{2}\right)$. We claim, that $d_{S^{2}, 2}^{7}\left(\operatorname{index}_{\mathbb{R}}(\mathcal{E})\right) \neq$ $0 \in H^{2}\left(S^{2}, \mathbb{R} / \mathbb{Z}\right)$.

We proceed as in 3.3.3. We consider a taming $\mathcal{E}_{t}$. It induces a perturbation $D_{t}(a)$ of the total operator $D(a)$ on $E$. We have again

$$
\left[\lim _{a \rightarrow 0} \eta^{0}\left(D_{t}(a)\right)\right]_{\mathbb{R} / \mathbb{Z}}=\left[\int_{S^{2}} \eta^{2}\left(\mathcal{E}_{t}\right)\right]_{\mathbb{R} / \mathbb{Z}}=d_{S^{2}, 2}^{7}\left(\operatorname{index}_{\mathbb{R}}(\mathcal{E})\right)\left(\left[S^{2}\right]\right) .
$$

Again, for sufficiently small $a$ the class $\left[\eta^{0}\left(D_{t}(a)\right)\right]_{\mathbb{R} / \mathbb{Z}}$ is independent of the adiabatic

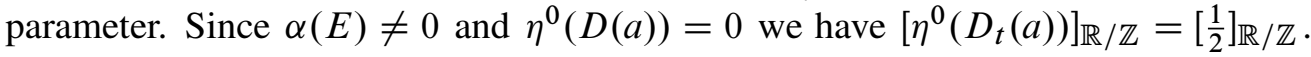
This implies the claim.

\section{Topological universal classes}

\subsection{Transgression of the Chern classes}

4.1.1 As proposed in 3.2.1 we shall understand $d_{B, 4 k+1}^{0}$ through its universal example. In the present section we start with the definition of this universal class. We will obtain an expression of this class in terms of familiar characteristic classes of real vector 
bundles. In Theorem 4.7 we show that the transformation $d_{B, 4 k+1}^{0}$ is indeed induced by the corresponding universal example. In Theorem 4.8 we will then identify $d_{B, 4 k+1-n}^{-n}$ in topological terms for all $n$. Then we will establish some vanishing results and more details about the topological side.

4.1.2 In the present section all spaces have distinguished base points and all maps are base point preserving. Let $O$ and $U$ be the direct limits of $O(n)$ and $U(n)$ induced by the embedding into the left upper corner. The embeddings $\mathbb{R}^{n} \hookrightarrow \mathbb{C}^{n}, n \in \mathbb{N}$, induce embeddings $O(n) \hookrightarrow U(n)$ and $c: O \hookrightarrow U$. The map $c$ induces the complexification transformation $c_{B}$. (see 2.2.1).

4.1.3 Let $E U \rightarrow B U$ be a universal bundle for $U$. We can consider $B O:=E U / O$ and obtain a bundle

$$
U / O \stackrel{i}{\rightarrow} B O \stackrel{p}{\rightarrow} B U
$$

with fiber $U / O$ over the base point of $B U$, compare (12) and (11) in B.2.1.

In the following, we use the transgression homomorphism for cohomology associated to this fibration (4). For the convenience of the reader, we have collected the main definitions and properties of transgression in general (with proofs) in Appendix A, and of transgression and cohomology of the spaces in the fibration $U / O \rightarrow B O \rightarrow B U$ in Lemma B.6 in Appendix B.

4.1.4 We will use the following definition.

Definition 4.1 We define the universal transgressed Chern classes

$$
d_{4 k+1}:=T\left(c_{4 k+2}^{\mathbb{Q}}\right) \in H^{4 k+1}(U / O, \mathbb{Q}),
$$

where $c_{4 k+2}^{\mathbb{Q}}$ is the image of the universal Chern class $c_{4 k+2}$ under the natural map

$$
H^{4 k+2}(B U, \mathbb{Z}) \rightarrow H^{4 k+2}(B U, \mathbb{Q}) .
$$

4.1.5 We now consider the following commutative diagram:

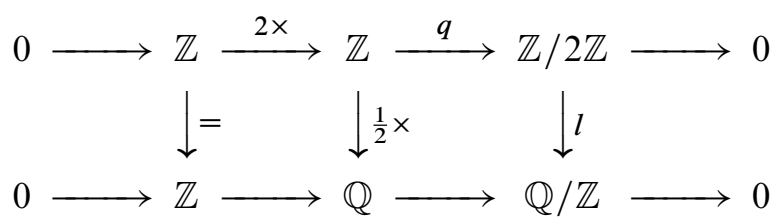

Definition 4.2 We define $\bar{d}_{4 k+1} \in H^{4 k+1}(U / O, \mathbb{Q} / \mathbb{Z})$ as the image of $d_{4 k+1}$ under the natural map $H^{4 k+1}(U / O, \mathbb{Q}) \rightarrow H^{4 k+1}(U / O, \mathbb{Q} / \mathbb{Z})$. 
4.1.6 The cohomology ring of $B O$ with coefficients in $\mathbb{Z} / 2 \mathbb{Z}$ is a polynomial ring

$$
\mathbb{Z} / 2 \mathbb{Z}\left[w_{1}, w_{2}, \ldots\right],
$$

where $w_{i} \in H^{i}(B O, \mathbb{Z} / 2 \mathbb{Z})$ are the universal Stiefel-Whitney classes. It is well known (see Milnor and Stasheff [12]) that

$$
\beta\left(w_{2 k} \cup w_{2 k+1}\right)=p^{*} c_{4 k+2},
$$

where $\beta$ is the cohomological Bockstein operator associated to the exact sequence of coefficients in the first row of (5). In particular, $2 p^{*} c_{4 k+2}=0 \in H^{4 k+2}(B O ; \mathbb{Z})$, as also stated in Appendix B.

4.1.7 Let $l_{*}: H^{4 k+1}(B O, \mathbb{Z} / 2 \mathbb{Z}) \rightarrow H^{4 k+1}(B O, \mathbb{Q} / \mathbb{Z})$ be induced by $l$ of (5). Note that by Lemma B.5 the map $l_{*}$ factors over the image of $\beta$.

Definition 4.3 We define

$$
\tilde{d}_{4 k+1}:=l_{*} u \in H^{4 k+1}(B O, \mathbb{Q} / \mathbb{Z}),
$$

where $u \in H^{4 k+1}(B O, \mathbb{Z} / 2 \mathbb{Z})$ is such that $\beta(u)=p^{*} c_{4 k+2}$.

Such an $u$ exists by (6) and $l_{*} u$ is independent of the choice since we have fixed $\beta(u)$.

4.1.8 Let $i: U / O \hookrightarrow B O$ be the inclusion.

Lemma 4.4 We have $i^{*} \tilde{d}_{4 k+1}=\bar{d}_{4 k+1}$. Moreover, $2 \tilde{d}_{4 k+1}=0$ and $2 \bar{d}_{4 k+1}=0$.

Proof The first assertion is a special case of Proposition A.12, where we use the equality $2 p^{*}\left(c_{4 k+2}\right)=0$. Note that the homomorphism $l$ is given by division by 2 .

The second assertion follows from the fact that $2 \tilde{d}_{4 k+1}=2 l_{*} u=l_{*}(2 u)=0$ for $u$ of Definition 4.3 , and $2 \bar{d}_{4 k+1}=i^{*}\left(2 \tilde{d}_{4 k+1}\right)=0$.

4.1.9 By (6) we have the following corollary.

Corollary 4.5 We have $\tilde{d}_{4 k+1}=l_{*}\left(w_{2 k} \cup w_{2 k+1}\right)$. 


\subsection{A topological description of $d_{B, 4 k+1}^{0}$}

4.2.1 In this subsection we pretend that $p: B O \rightarrow B U$ is a smooth fiber bundle. To be precise, we should replace this bundle by an $N$-equivalent finite-dimensional smooth bundle for $N$ sufficiently large.

Let $W_{\mathbb{R}}^{+} \rightarrow B O$ be the universal bundle. Then $p: B O \rightarrow B U$ classifies its complexification, ie if $W^{+} \rightarrow B U$ is the universal bundle over $B U$, then we have an isomorphism $W_{\mathbb{R}}^{+} \otimes_{\mathbb{R}} \mathbb{C} \cong p^{*} W^{+}$which induces a real structure $Q$ (complex conjugation) on $p^{*} W^{+}$. We can assume that $W_{\mathbb{R}}^{+}$comes with a metric $h^{W_{\mathbb{R}}^{+}}$, and we choose a connection $\nabla^{W_{\mathbb{R}}^{+}}$. We set $\mathbf{W}_{\mathbb{R}}^{+}:=\left(W_{\mathbb{R}}^{+}, \nabla_{\mathbb{R}}^{+}, h^{W_{\mathbb{R}}^{+}}\right)$and let $\mathbf{W}^{+}$be its complexification.

4.2.2 We now consider the $\mathbb{Z} / 2 \mathbb{Z}$-graded bundle $\mathbf{W}:=\mathbf{W}^{+} \oplus \mathbf{W}^{-}$with $\mathbf{W}^{-}:=\overline{\mathbf{W}}^{+}$. It admits an odd unitary selfadjoint (not necessary parallel) automorphism

$$
R:=\left(\begin{array}{cc}
0 & Q \\
Q & 0
\end{array}\right)
$$

We form the geometric families $\mathcal{G}^{ \pm}$on $B O$ with underlying fiber bundle id: $B \rightarrow B$ and twisting bundles $p^{*} \mathbf{W}^{ \pm}$. Then the family $\mathcal{G}=\mathcal{G}^{+} \cup_{B} \mathcal{G}^{-}$admits a taming $\mathcal{G}_{t}$ induced by $R$. The associated $\eta$-form satisfies $d \eta\left(\mathcal{G}_{t}\right)=\Omega(\mathcal{G})=\operatorname{ch}\left(\nabla^{p^{*} W}\right)$. By construction we have $\operatorname{ch}_{4 k+2}\left(\nabla^{p^{*} W}\right)=2 p^{*} \mathbf{c h}_{4 k+2}\left(\nabla^{W^{+}}\right)$and $\operatorname{ch}_{4 k}\left(\nabla^{p^{*} W}\right)=0$ (compare 2.1.18).

4.2.3 Let $i: U / O \rightarrow B O$ be the inclusion of the fiber. Then

$$
d i^{*} \eta^{4 k+1}\left(\mathcal{G}_{t}\right)=2\left(i^{*} \circ p^{*}\right) \mathbf{c h}_{4 k+2}\left(\nabla^{W^{+}}\right)=0
$$

since $p \circ i$ is a constant map. Thus we can consider the class

$$
\left[i^{*} \eta^{4 k+1}\left(\mathcal{G}_{t}\right)\right] \in H^{4 k+1}(U / O, \mathbb{R}) .
$$

4.2.4 Let $d_{4 k+1}^{\mathbb{R}} \in H^{4 k+1}(U / O, \mathbb{R})$ be the image of $d_{4 k+1} \in H^{4 k+1}(U / O, \mathbb{Q})$ under the natural map induced by the map of coefficients $r: \mathbb{Q} \hookrightarrow \mathbb{R}$.

Lemma 4.6 We have

$$
\left[i^{*} \eta^{4 k+1}\left(\mathcal{G}_{t}\right)\right]=\frac{2 d_{4 k+1}^{\mathbb{R}}}{(2 k) !}
$$

Proof The proof follows from the fact that in the smooth situation there is the alternative description of the transgression $T^{\mathbb{R}}: H^{4 k+2}(B U, \mathbb{R}) \rightarrow H^{4 k+1}(U / O, \mathbb{R})$ given in Proposition A.11. Let $x \in H^{4 k+2}(B U, \mathbb{R})$ be represented by a closed form 
$X \in \mathcal{A}^{4 k+2}(B U)$. Then there is some form $Y \in \mathcal{A}^{4 k+1}(B O)$ such that $d Y=p^{*} X$. The class $T^{\mathbb{R}}(x) \in H_{d R}^{4 k+1}(U / O, \mathbb{R})$ is then represented by $i^{*} Y \in \mathcal{A}^{4 k+1}(U / O)$.

In our case

$$
x=2 \mathbf{c h}_{4 k+2}^{\mathbb{R}}\left(\left[W^{+}\right]\right), \quad X=2 \mathbf{c h}_{4 k+2}\left(\nabla^{W^{+}}\right), \quad Y=\eta^{4 k+1}\left(\mathcal{G}_{t}\right)
$$

so that

$$
\left[i^{*} \eta^{4 k+1}\left(\mathcal{G}_{t}\right)\right]=T^{\mathbb{R}}\left(2 \mathbf{c h}_{4 k+2}^{\mathbb{R}}\left(\left[W^{+}\right]\right)\right)
$$

Note that

$$
\operatorname{ch}_{4 k+2}\left(\left[W^{+}\right]\right)=\frac{1}{(2 k) !} c_{4 k+2}^{\mathbb{Q}}+\text { decomposable classes, }
$$

where for reasons of degree each decomposable summand contains at least one factor $c_{4 l+2}$ which is transgressive by Table 1 . Hence by Proposition A.10

$$
T\left(2 \mathbf{c h}_{4 k+2}\left(\left[W^{+}\right]\right)\right)=\frac{2}{(2 k) !} T\left(c_{4 k+2}\right)=\frac{2 d_{4 k+1}}{(2 k) !} .
$$

This implies the assertion since $T^{\mathbb{R}} \circ r_{*}=r_{*} \circ T$ by Lemma A.7.

4.2.5 We now consider a manifold $B$ and $x \in \widetilde{K O^{0}}(B)$. Let $X: B \rightarrow B O$ be a classifying map for $x$. We assume that $x \in U_{\infty}^{0}(B)$. Then we can assume that $X$ factors through the inclusion $i: U / O \rightarrow B O$, ie without loss of generality we can assume that $X: B \rightarrow U / O$.

We define $\bar{d}_{4 k+1}^{\mathbb{R}} \in H^{4 k+1}(B, \mathbb{R} / \mathbb{Z})$ as the image of $d_{4 k+1}^{\mathbb{R}}$ under the map of coefficients $\mathbb{R} \rightarrow \mathbb{R} / \mathbb{Z}$, or equivalently, as the image of $\bar{d}_{4 k+1}$ under the map of coefficients $\mathbb{Q} / \mathbb{Z} \rightarrow \mathbb{R} / \mathbb{Z}$. We now come to the main result of this subsection.

Theorem 4.7 $d_{B, 4 k+1}^{0}(x)=X^{*} \bar{d}_{4 k+1}^{\mathbb{R}}$.

Proof Let $x$ be of the form $\left[V_{\mathbb{R}}^{+}\right]-\left[V_{\mathbb{R}}^{-}\right]$, where $V_{\mathbb{R}}^{-}:=\mathbb{R}^{N} \times B$ is trivial. Then we have an isomorphism $V_{\mathbb{R}}^{+} \cong X^{*} W_{\mathbb{R}}^{+}$. The metric $h^{W_{\mathbb{R}}^{+}}$and the connection $\nabla^{W_{\mathbb{R}}^{+}}$ induce a metric $h^{V_{\mathbb{R}}^{+}}$and a connection $\nabla^{V_{\mathbb{R}}^{+}}$on $V_{\mathbb{R}}^{+}$. In this way we obtain a geometric bundle $\mathbf{V}_{\mathbb{R}}^{+}=\left(V_{\mathbb{R}}^{+}, \nabla_{\mathbb{R}^{+}}^{+}, h_{\mathbb{R}}^{+}\right)$. Furthermore, we equip $V_{\mathbb{R}}^{-}$with the canonical geometry and get $\mathbf{V}_{\mathbb{R}}^{-}$.

Set $\mathbf{V}^{ \pm}:=\mathbf{V}_{\mathbb{R}}^{ \pm} \otimes \mathbb{C}$ and consider the $\mathbb{Z} / 2 \mathbb{Z}$-graded bundle $\mathbf{V}:=\mathbf{V}^{+} \oplus \mathbf{V}^{-}$. Because $[V]=0$ in $K^{0}(B)$ we can choose a unitary odd selfadjoint (not necessary parallel) automorphism $U$ of $V$.

We form the geometric families $\mathcal{H}^{ \pm}$over $B$ with underlying bundle id: $B \rightarrow B$ and twisting bundle $\mathbf{V}^{ \pm}$. Furthermore we define $\mathcal{H}:=\mathcal{H}^{+} \cup_{B}\left(\mathcal{H}^{-}\right)^{\text {op }}$. Then we have 
$\operatorname{index}_{\mathbb{R}}(\mathcal{H})=x$ and $d_{4 k+1, B}^{0}(x)=\widehat{c}_{4 k+2}(\mathcal{H})$. The isomorphism $U$ induces a taming $\mathcal{H}_{t}$. By Corollary 3.7 we thus have

$$
\widehat{c}_{4 k+2}(\mathcal{H})=\left[(2 k) ! \eta^{4 k+1}\left(\mathcal{H}_{t}\right)\right]_{\mathbb{R} / \mathbb{Z}} \in H^{4 k+1}(B, \mathbb{R} / \mathbb{Z}) .
$$

We now consider the bundle $\tilde{\mathbf{V}}:=\mathbf{V}^{+} \oplus \overline{\mathbf{V}}^{-} \oplus \overline{\mathbf{V}}^{+} \oplus \mathbf{V}^{-}$with the $\mathbb{Z} / 2 \mathbb{Z}$-grading $\operatorname{diag}(1,1,-1,-1)$ and the two odd automorphisms

$$
\tilde{U}:=\left(\begin{array}{cccc}
0 & 0 & 0 & U^{-} \\
0 & 0 & \bar{U}^{+} & 0 \\
0 & \bar{U}^{-} & 0 & 0 \\
U^{+} & 0 & 0 & 0
\end{array}\right), \quad \widetilde{R}:=\left(\begin{array}{cccc}
0 & 0 & R^{+} & 0 \\
0 & 0 & 0 & R^{-} \\
R^{+} & 0 & 0 & 0 \\
0 & R^{-} & 0 & 0
\end{array}\right),
$$

where $R^{ \pm}$is the $\mathbb{C}$-linear isomorphism between $V_{\mathbb{R}}^{ \pm} \otimes \mathbb{C}$ and its complex conjugate given by complex conjugation.

Note that $[\widetilde{R}, \tilde{U}]=0$. The bundle $\tilde{\mathbf{V}}$ gives rise to a geometric family $\tilde{\mathcal{H}}=\widetilde{\mathcal{H}}^{+} \cup_{B}$ $\left(\widetilde{\mathcal{H}}^{-}\right)^{\text {op }}$, where the underlying fiber bundle of $\widetilde{\mathcal{H}}^{ \pm}$is again id: $B \rightarrow B$, and the twisting bundles are $\tilde{\mathbf{V}}^{ \pm}$. For each $\alpha \in[0, \pi / 2]$ the operator

$$
\cos (\alpha) \tilde{U}+\sin (\alpha) \tilde{R}
$$

defines a taming $\tilde{\mathcal{H}}_{t_{\alpha}}$. The family $\left(\tilde{\mathcal{H}}_{t_{\alpha}}\right)_{\alpha}$ defines a taming $\hat{\mathcal{H}}_{t}$ of $\hat{\mathcal{H}}:=\operatorname{pr}_{B}^{*} \tilde{\mathcal{H}}$ over $[0, \pi / 2] \times B$. A computation shows that $d \eta\left(\hat{\mathcal{H}}_{t}\right)=\operatorname{pr}_{B}^{*} \operatorname{ch}(\nabla \widetilde{V})=0$. We conclude the following equality of de Rham cohomology classes

$$
\left[\eta^{4 k+1}\left(\tilde{\mathcal{H}}_{t_{0}}\right)\right]=\left[\eta^{4 k+1}\left(\tilde{\mathcal{H}}_{t_{\pi / 2}}\right)\right] .
$$

An inspection of the definitions shows that

$$
\begin{aligned}
\eta^{4 k+1}\left(\tilde{\mathcal{H}}_{t_{0}}\right) & =2 \eta^{4 k+1}\left(\mathcal{H}_{t}\right) \\
\eta^{4 k+1}\left(\tilde{\mathcal{H}}_{t_{\pi / 2}}\right) & =\left(X^{*} \circ i^{*}\right) \eta^{4 k+1}\left(\mathcal{G}_{t}\right) .
\end{aligned}
$$

We conclude with Lemma 4.6 that

$$
\widehat{c}_{4 k+2}(\mathcal{H})=X^{*}\left[i^{*} \frac{(2 k) !}{2} \eta^{4 k+1}\left(\mathcal{G}_{t}\right)\right]_{\mathbb{R} / \mathbb{Z}}=X^{*} \bar{d}_{4 k+1}^{\mathbb{R}} .
$$

\subsection{The topological interpretation of $d_{B, 4 k+1-n}^{-n}$}

4.3.1 Recall that the classifying space of $K O^{-n}$ is $\Omega^{n} B O$. In view of the fibration

$$
\Omega^{n}(U / O) \rightarrow \Omega^{n} B O \rightarrow \Omega^{n} B U
$$


we see that the classifying map $X: B \rightarrow \Omega^{n} B O$ of an element $x \in U_{\infty}^{-n}(B)$ factors (up to homotopy) over $\Omega^{n}(U / O)$, since then the composition

$$
B \stackrel{X}{\rightarrow} \Omega^{n} B O \rightarrow \Omega^{n} B U
$$

is null homotopic.

Let $\Omega^{n}: H^{4 k+1}(U / O, \mathbb{R}) \rightarrow H^{4 k+1-n}\left(\Omega^{n}(U / O), \mathbb{R}\right)$ be the $n$-fold iteration of the loop map introduced in Definition A.1.

Theorem 4.8 We have

$$
d_{B, 4 k+1-n}^{-n}(x)=X^{*}\left[\frac{(m-1) !}{(2 k) !} \Omega^{n} d_{4 k+1}^{\mathbb{R}}\right]_{\mathbb{R} / \mathbb{Z}}=X^{*}\left[\frac{(m-1) !}{(2 k) !} \Omega^{n} T\left(c_{4 k+2}^{\mathbb{R}}\right)\right]_{\mathbb{R} / \mathbb{Z}},
$$

where $m$ is determined by $2 m=4 k+3-n$ or $2 m=4 k+2-n$.

Proof We employ Corollary 3.7. Let $\hat{x} \in \widetilde{K O}^{0}\left(\Sigma^{n} B\right)$ correspond to $x \in K O^{-n}(B)$ under the identification $K O^{-n}(B) \cong \widetilde{K O}^{0}\left(\Sigma^{n} B\right)$. Let $\tilde{x} \in \widetilde{K O}^{0}\left(S^{n} \times B\right)$ be the pullback of $\hat{x}$ under the natural map $S^{n} \times B \rightarrow \Sigma^{n} B$.

Note that the classifying map $Y: \Sigma^{n} B \rightarrow U / O$ of $\hat{x}$ is the adjoint of $X: B \rightarrow$ $\Omega^{n}(U / O)$, and that the composition $\tilde{X}: S^{n} \times B \rightarrow U / O$ of the projection $S^{n} \times B \rightarrow$ $\Sigma^{n} B$ and $Y$ is the classifying map of $\tilde{x}$.

Then we have

$$
d_{B, 4 k+1-n}^{-n}(x)=\left[(m-1) ! \int_{S^{n} \times B / B} \eta^{4 k+1}\left(\mathcal{H}_{t}\right)\right]_{\mathbb{R} / \mathbb{Z}},
$$

where $\mathcal{H}_{t}$ is constructed as in the proof of Theorem 4.7. In that proof we have also shown that

$$
\left[\eta^{4 k+1}\left(\mathcal{H}_{t}\right)\right]=\left[\frac{1}{2}\left(\tilde{X}^{*} \circ i^{*}\right) \eta^{4 k+1}\left(\mathcal{G}_{t}\right)\right] .
$$

We now apply Lemma 4.6 in order to conclude that

$$
\left[\eta^{4 k+1}\left(\mathcal{H}_{t}\right)\right]=\frac{\tilde{X}^{*} d_{4 k+1}^{\mathbb{R}}}{(2 k) !}
$$

Thus

$$
d_{B, 4 k+1-n}^{-n}(x)=\left[\frac{(m-1) !}{(2 k) !} \int_{S^{n} \times B / B} \tilde{X}^{*} d_{4 k+1}^{\mathbb{R}}\right]_{\mathbb{R} / \mathbb{Z}} .
$$

The assertion now follows from the general fact that for any $z \in H^{4 k+1}(U / O, \mathbb{R})$ we have

$$
\int_{S^{n} \times B / B} \tilde{X}^{*} z=\Sigma^{n} Y^{*} z=X^{*} \Omega^{n} z
$$


where $\Sigma$ is the suspension isomorphism. For the first equality we use that integration over the fiber essentially is the suspension isomorphism in the above construction. The second equality is a special case of the relation between suspension and loop homomorphism proved in Lemma A.6.

4.3.2 Theorem 4.7 and Theorem 4.8 give a topological description of the value of $d_{B, 4 k+1-n}^{-n}(x)$ only under the additional assumption that $x \in U_{\infty}^{-n}(B) \subset U_{4 k+2-n}^{-n}(B)$. In order to see that this determines $d_{B, 4 k+1-n}^{-n}$ completely we argue as follows.

Let $x \in U_{4 k+2-n}^{-n}(B)$. Then the cohomology class $d_{B, 4 k+1-n}^{-n}(x)$ of degree $4 k+1-n$ is determined by its restriction $d_{B, 4 k+1-n}^{-n}(x)_{\mid B^{4 k+1-n}}$ to the $(4 k+1-n)$-skeleton

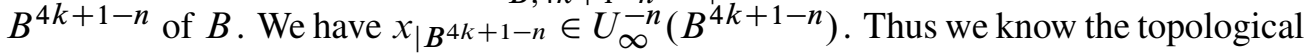
description of $d_{B^{4 k+1-n}, 4 k+1-n}^{-n}\left(x_{\mid B^{4 k+1-n}}\right)$, which equals $d_{B, 4 k+1-n}^{-n}(x)_{\mid B^{4 k+1-n}}$ by naturality.

\subsection{Explicit calculation of the universal class}

Theorem 4.8 does give a topological interpretation of our invariant 3.1. However, we want to be even more precise and explicitly compute the corresponding universal cohomology class

$$
\begin{aligned}
{\left[\frac{(m-1) !}{(2 k) !} \Omega^{n} d_{4 k+1}\right]_{\mathbb{Q} / \mathbb{Z}} } & \\
= & {\left[\frac{(m-1) !}{(2 k) !} \Omega^{n} T\left(c_{4 k+2}^{\mathbb{Q}}\right)\right]_{\mathbb{Q} / \mathbb{Z}} \in H^{4 k+1-n}\left(\Omega^{n} U / O, \mathbb{Q} / \mathbb{Z}\right), }
\end{aligned}
$$

where $m$ is determined by $2 m=4 k+3-n$ or $2 m=4 k+2-n$. In particular, we will show that for half of the parameters $n(\bmod (8))$ this class vanishes

We will make use of many of the results about the cohomology of $B O, B U$ and their loop spaces collected in Appendix B.

4.4.1 Consider the map of fibrations

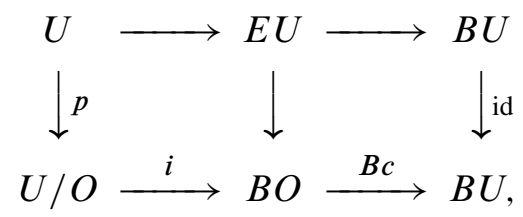

where the upper row is the universal principal $U$-bundle, and the lower row is obtained from the upper by dividing out the subgroup $O$. 
4.4.2 By Lemma B.6, $c_{4 k+2}^{\mathbb{Q}} \in H^{4 k+2}(B U, \mathbb{Q})$ is transgressive in the second (and of course also in the first) fibration. We have to compute the transgression $T\left(c_{4 k+2}\right) \in$ $H^{4 k+1}(U / O, \mathbb{Q})$. To do this, we observe that the upper fibration is the path space fibration, and therefore by Lemma A.4 the transgression $T_{U}$ of this fibration coincides with the loop homomorphism. By Theorem B.4 we obtain $T_{U}\left(c_{4 k+2}\right)=c_{4 k+1}$ (even in integral cohomology). Moreover, transgression is natural, therefore

$$
p^{*}\left(T\left(c_{4 k+2}^{\mathbb{Q}}\right)\right)=T_{U}\left(c_{4 k+2}^{\mathbb{Q}}\right)=c_{4 k+1}^{\mathbb{Q}} \in H^{*}(U, \mathbb{Q}) .
$$

By Theorem B.2, Theorem B.3 and Table $1, p^{*}: H^{*}(U / O, \mathbb{Q}) \rightarrow H^{*}(U, \mathbb{Q})$ is injective and $p^{*}\left(a_{4 k+1} / 2\right)=c_{4 k+1}$.

Notation 4.9 In order to avoid an inflationary appearance of the exponent $\mathbb{Q}$ from now on we will use the same symbol for an integral cohomology class and its image in rational cohomology. It will be clear from the context which meaning the symbol has.

Consequently (with the new convention 4.9 ) we can write

$$
T\left(c_{4 k+2}\right)=\frac{1}{2} a_{4 k+1} \in H^{4 k+1}(U / O ; \mathbb{Q}) .
$$

4.4.3 Our next goal is the calculation of

$$
\Omega^{n} T\left(c_{4 k+2}\right)=\Omega^{n}\left(\frac{1}{2} a_{4 k+1}\right) \in H^{4 k+1-n}\left(\Omega^{n}(U / O), \mathbb{Q}\right) .
$$

We consider the fibration

$$
\Omega^{n}(U / O) \rightarrow \Omega^{n} B O \rightarrow \Omega^{n} B U
$$

which is the $n$-fold loop of the fibration considered above.

4.4.4 In the following, we use the Bott periodicity maps to identify $\Omega^{n}(U / O)$ with the spaces listed in Theorem B.1:

\begin{tabular}{l|llllllll}
$n$ & 0 & 1 & 2 & 3 & 4 & 5 & 6 & 7 \\
\hline$\Omega^{n}(U / O)$ & $\mathrm{U} / \mathrm{O}$ & $B O \times \mathbb{Z}$ & $\mathrm{O}$ & $\mathrm{O} / \mathrm{U}$ & $\mathrm{U} / \mathrm{Sp}$ & $B S p \times \mathbb{Z}$ & $\mathrm{Sp}$ & $\mathrm{Sp} / \mathrm{U}$
\end{tabular}

4.4.5 Unfortunately, our knowledge about the map $\Omega^{n}$ is not complete enough to calculate $\Omega^{n}\left(T c_{4 k+2}\right)$ directly. We use the following trick:

Using that map $\alpha: U / O \rightarrow U$ (compare Section B.2, Item (16)) we have $\frac{1}{2} a_{4 k+1}=$ $\alpha^{*} \frac{1}{2} c_{4 k+1}$. Therefore,

$$
\Omega^{n}\left(\frac{1}{2} a_{4 k+1}\right)=\left(\Omega^{n} \alpha\right)^{*} \Omega^{n}\left(\frac{1}{2} c_{4 k+1}\right) .
$$


4.4.6 We shall first compute $\Omega^{n}\left(c_{4 k+1}\right)$. By Theorem B.4

$$
\Omega\left(c_{4 k+1}\right)=(2 k) ! \mathbf{c h}_{4 k} .
$$

Note that $\Omega B U=\Omega(B U \times \mathbb{Z})$ so that we can iterate the argument.

Next, $(2 k) ! \mathbf{c h}_{4 k}=2 k \cdot c_{4 k}+$ decomposable. We conclude that

$$
\Omega\left((2 k) ! \mathbf{c h}_{4 k}\right)=2 k \Omega\left(c_{4 k}\right)=2 k c_{4 k-1} .
$$

4.4.7 Now, an easy induction allows us to compute $\Omega^{n}\left(c_{4 k+1}\right)$ for each $n \in \mathbb{N}$. However, because of the factors appearing in our formulas, we really have to study $((m-1) ! /(2 k) !) \Omega^{n}\left(c_{4 k+1}\right)$, where $m=2 k+1-n / 2$ if $n$ is even, and $m=2 k+$ $1-(n-1) / 2$ if $n$ is odd. In the induction, this factor cancels the factors (like $(2 k))$ which show up in the calculations above, and we get (with $m$ depending on $n$ and $k$ as above)

$$
\Omega^{n}\left(\frac{(m-1) !}{(2 k) !} c_{4 k+1}\right)= \begin{cases}c_{4 k+1-n} & n \text { even } \\ (2 k-(n-1) / 2) ! \mathbf{c h}_{4 k+1-n} & n \text { odd } .\end{cases}
$$

4.4.8 From this and the calculation of the map in cohomology induced by the map $\Omega^{n} \alpha: \Omega^{n}(U / O) \rightarrow \Omega^{n} U$, we read off the cohomology classes we are interested in. Since we are really only interested in the image of the class under the map in cohomology induced by the map of coefficients $\mathbb{Q} \rightarrow \mathbb{Q} / \mathbb{Z}$, we obtain the following list:

\section{Theorem 4.10}

$$
\begin{aligned}
& {\left[\frac{(m-1) !}{(2 k) !} T\left(c_{4 k+2}\right)\right]_{\mathbb{Q} / \mathbb{Z}}=\left[\frac{1}{2} a_{4 k+1}\right]_{\mathbb{Q} / \mathbb{Z}} \in H^{4 k+1}(U / O ; \mathbb{Q} / \mathbb{Z})} \\
& {\left[\Omega\left(\frac{(m-1) !}{(2 k) !} T\left(c_{4 k+2}\right)\right)\right]_{\mathbb{Q} / \mathbb{Z}}= \begin{cases}{\left[\frac{1}{2} \mathbf{c h}_{0}\right]_{\mathbb{Q} / \mathbb{Z}} \in H^{0}(B O \times \mathbb{Z}, \mathbb{Q} / \mathbb{Z})} & 4 k+1=n \\
0 \in H^{4 k}(B O \times \mathbb{Z} ; \mathbb{Q} / \mathbb{Z}) & 4 k+1>n\end{cases} } \\
& {\left[\Omega^{2}\left(\frac{(m-1) !}{(2 k) !} T\left(c_{4 k+2}\right)\right)\right]_{\mathbb{Q} / \mathbb{Z}}=0 \in H^{4 k-1}(O, \mathbb{Q} / \mathbb{Z})} \\
& {\left[\Omega^{3}\left(\frac{(m-1) !}{(2 k) !} T\left(c_{4 k+2}\right)\right)\right]_{\mathbb{Q} / \mathbb{Z}}=0 \in H^{4 k-2}(O / U, \mathbb{Q} / \mathbb{Z})} \\
& {\left[\Omega^{4}\left(\frac{(m-1) !}{(2 k) !} T\left(c_{4 k+2}\right)\right)\right]_{\mathbb{Q} / \mathbb{Z}}=0 \in H^{4 k-3}(U / S p, \mathbb{Q} / \mathbb{Z})} \\
& {\left[\Omega^{5}\left(\frac{(m-1) !}{(2 k) !} T\left(c_{4 k+2}\right)\right)\right]_{\mathbb{Q} / \mathbb{Z}}=0 \in H^{4 k-4}(B S p \times \mathbb{Z}, \mathbb{Q} / \mathbb{Z})}
\end{aligned}
$$




$$
\begin{gathered}
{\left[\Omega^{6}\left(\frac{(m-1) !}{(2 k) !} T\left(c_{4 k+2}\right)\right)\right]_{\mathbb{Q} / \mathbb{Z}}=\left[\frac{1}{2} y_{4 k-5}\right]_{\mathbb{Q} / \mathbb{Z}} \in H^{4 k-5}(S p, \mathbb{Q} / \mathbb{Z})} \\
{\left[\Omega^{7}\left(\frac{(m-1) !}{(2 k) !} T\left(c_{4 k+2}\right)\right)\right]_{\mathbb{Q} / \mathbb{Z}}=\left[\frac{1}{2}\left(c_{4 k-6}+c_{2} c_{4 k-8}+\cdots+c_{2 k-4} c_{2 k-2}\right)\right]_{\mathbb{Q} / \mathbb{Z}}} \\
\in H^{4 k-6}(S p / U, \mathbb{Q} / \mathbb{Z})
\end{gathered}
$$

For $n \geq 8$, the answer can be read off from the list by reduction mod 8 by Bott periodicity.

In particular, the natural transformation $d_{B, 4 k+1-n}^{-n}$ vanishes for $n$ congruent to 2, 3, 4, $5 \bmod 8$, and, if $k>0$, also for $n \equiv 1(\bmod 8)$. In the other cases, since the universal classes are nontrivial, there are nontrivial examples.

Proof As observed above, we simply have to take the cohomology classes on the right-hand side of Equation (8), divide them by 2, and then apply the map from rational cohomology to cohomology with coefficients in $\mathbb{Q} / \mathbb{Z}$. Finally through $\Omega^{n} \alpha$ we pull back the result to $\Omega^{n}(U / O)$. In this step we use the results of Section B.2.

Note first that $x_{n}:=c_{4 k+1-n}\left(n\right.$ even) and $x_{n}:=(2 k-(n-1) / 2) ! \mathbf{c h}_{4 k+1-n}(n$ odd $)$ belong to the integral lattice in rational cohomology. Therefore $\left[\frac{1}{2}\left(\Omega^{n} \alpha\right)^{*} \Omega^{n} x_{n}\right]_{\mathbb{R} / \mathbb{Z}}=$ 0 if $\frac{1}{2}\left(\Omega^{n} \alpha\right)^{*}$ preserves the integral lattices. This is the case whenever $\Omega^{n} \alpha$ maps the Chern classes to twice a generator of the integral cohomology, ie if $\Omega^{n}(U / O)$ equals $O, O / U$, or $U / S p$ by the Table 1 . This observation accounts for the zeros for $n=2,3,4$ in the theorem.

Because of Proposition A.8

$$
\left[T\left(\Omega^{5}\left(\frac{(m-1) !}{(2 k) !} c_{4 k+2}\right)\right)\right]_{\mathbb{Q} / \mathbb{Z}}=\left[\Omega T\left(\Omega^{4}\left(\frac{(m-1) !}{(2 k) !} c_{4 k+2}\right)\right)\right]_{\mathbb{Q} / \mathbb{Z}}=0,
$$

we obtain the zero for $n=5$. We now discuss the case $n=1$. We have $\left[T\left(c_{4 k+2}\right)\right]_{\mathbb{Q} / \mathbb{Z}}=$ $l_{*}\left(w_{2 k} \cup w_{2 k+1}\right)$, where $l_{*}$ is induced by the map of coefficients

$$
\mathbb{Z} / 2 \mathbb{Z} \rightarrow \mathbb{Q} / 2 \mathbb{Z} \stackrel{\cdot 1 / 2}{\longrightarrow} \mathbb{Q} / \mathbb{Z}
$$

(compare 4.1.5 and Corollary 4.5). If $k>0$, then $\Omega\left(w_{2 k} \cup w_{2 k+1}\right)=0$ since the loop map is applied to a decomposable class. Note that $(m-1) ! /(2 k) !=1$ in this case. Thus

$$
\left[\Omega\left(\frac{(m-1) !}{(2 k) !} T\left(c_{4 k+2}\right)\right)\right]_{\mathbb{Q} / \mathbb{Z}}=l_{*} \Omega\left(w_{2 k} \cup w_{2 k+1}\right)=0
$$

for $k>0$.

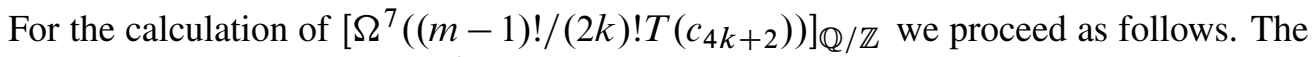
class $(2 k-3) ! \mathbf{c h}_{4 k-6} \in H^{4 k-6}(S p / U, \mathbb{Q})$ belongs to the integral lattice. In fact, if 
we write $(2 k-3) ! \mathbf{c h}_{4 k-6}=\sigma_{4 k-6}\left(c_{2}, c_{4}, \ldots, c_{4 k-6}\right)$ with the Newton polynomial $\sigma_{4 k-6}$, then the right-hand side can be interpreted as an integral cohomology class in $H^{4 k-6}(S p / U, \mathbb{Z})$. We now have

$$
l_{*}\left[\sigma_{4 k-6}\left(c_{2}, c_{4}, \ldots, c_{4 k-6}\right)\right]_{\mathbb{Z} / 2 \mathbb{Z}}=\left[\Omega^{7}\left(\frac{(m-1) !}{(2 k) !} T\left(c_{4 k+2}\right)\right)\right]_{\mathbb{Q} / \mathbb{Z}} .
$$

The cohomology $H^{4 k-6}(S p / U, \mathbb{Z} / 2 \mathbb{Z})$ is an exterior algebra generated by $\left[c_{2}\right]_{\mathbb{Z} / 2 \mathbb{Z}}$, $\left[c_{4}\right]_{Z / 2 \mathbb{Z}}, \ldots$ Considered-in this algebra-the Newton polynomial

$$
\begin{aligned}
& \sigma_{4 k-6}\left(c_{2}, c_{4}, \ldots, c_{4 k-6}\right) \\
& =-(2 k-3) \sum_{\substack{i_{1}+2 i_{2}+\cdots+(2 k-3) i_{2 k-3} \\
=2 k-3}}(-1)^{i_{1}+\cdots+i_{2 k-3}} \frac{\left(i_{1}+\cdots+i_{2 k-3}-1\right) !}{i_{1} ! \ldots i_{2 k-3} !} c_{2}^{i_{1}} \ldots c_{4 k-6}^{i_{2 k-3}} .
\end{aligned}
$$

It simplifies considerably and gives exactly the expression asserted, if we use that

$$
l_{*}\left[c_{2 l}\right]_{\mathbb{Z} / 2 \mathbb{Z}}=\left[\frac{1}{2} c_{2 l}\right]_{\mathbb{Q} / \mathbb{Z}} .
$$

\subsection{The relation with ordinary characteristic classes}

4.5.1 Let us consider the fibration

$$
\Omega^{n}(U / O) \stackrel{\Omega^{n} i}{\rightarrow} \Omega^{n} B O \rightarrow \Omega^{n} B U
$$

We have constructed and calculated the universal cohomology class

$$
\left[\Omega^{n}\left(\frac{(m-1) !}{(2 k) !} T\left(c_{4 k+2}\right)\right)\right]_{\mathbb{Q} / \mathbb{Z}} \in H^{4 k+1-n}\left(\Omega^{n}(U / O), \mathbb{Q} / \mathbb{Z}\right) .
$$

If this class would be of the form $\left(\Omega^{n} i\right)^{*} u$ for some $u \in H^{4 k+1-n}\left(\Omega^{n} B O, \mathbb{Q} / \mathbb{Z}\right)$, then the invariant $d_{B, 4 k+1-n}^{-n}(x), x \in U_{4 k+1-n}^{-n}(B)$, could be expressed in terms of familiar characteristic classes of the element $x \in K O^{-n}(B)$.

4.5.2 In the case $n=0$ we indeed have

$$
\left[\Omega^{n}\left(\frac{(m-1) !}{(2 k) !} T\left(c_{4 k+2}\right)\right)\right]_{\mathbb{Q} / \mathbb{Z}}=\left[T\left(c_{4 k+2}\right)\right]_{\mathbb{Q} / \mathbb{Z}}=i^{*} l_{*}\left(w_{2 k} \cup w_{2 k+1}\right)
$$

(above we have written $w_{l}$ for $i^{*} w_{l}$ in order to save notation, but in the current discussion it makes sense not to omit $\left.i^{*}\right)$. In particular we can extend $d_{B, 4 k+1}^{0}$ to all of $K O^{0}(B)$ by setting $d_{B, 4 k+1}^{0}(x):=l_{*}\left(w_{2 k} \cup w_{2 k+1}\right)$. 
4.5.3 In the case $n=1$ and $4 k=0$ it is obvious that the class comes from $\Omega^{1} B O$. As we have seen in Section 3.3.2, if $x \in K O^{-1}(X)$ is represented by a family of anti-selfadjoint real Fredholm operators $\left(F_{b}\right)_{b \in B}$, then $d_{B, 0}^{0}(x)$ is represented by the locally constant $\mathbb{R} / \mathbb{Z}$-valued function $b \mapsto\left[\frac{1}{2} \operatorname{dim} \operatorname{ker} F_{b}\right]_{\mathbb{R} / \mathbb{Z}}$

4.5.4 In the case $n=6$ the class

$$
\left[\Omega^{6}\left(\frac{(m-1) !}{(2 k) !} T\left(c_{4 k+2}\right)\right)\right]_{\mathbb{Q} / \mathbb{Z}}=\left[\Omega^{6}\left(\frac{1}{2 k(2 k-1)(2 k-2)} T\left(c_{4 k+2}\right)\right)\right]_{\mathbb{Q} / \mathbb{Z}}
$$

definitely is not a pullback from $\Omega^{6} B O=S p / U$. In fact $H^{*}(S p / U, \mathbb{Q} / \mathbb{Z})$ is concentrated in even degrees, while our class is of odd degree. We see that in this case our invariant $d_{B, 4 k-5}^{-6}$ is more exotic and therefore more interesting. Unfortunately, we haven't been able to produce simple examples of nontriviality for this invariant in Section 3.3.

4.5.5 For $n=7$, the map $S p / U \rightarrow U / O$ induces a surjection in cohomology with $\mathbb{Z} / 2 \mathbb{Z}$-coefficients by Lemma B.7. Therefore, all our classes pull back from $U / O$.

\subsection{Extendibility}

4.6.1 Given $x \in K O^{-n}(B)$, in order to define $d_{4 k+1-n}^{-n}(x)$ using topology we had to assume that $x \in U_{\infty}^{-n}(B)$. Our analytic definition however works under the weaker condition that $x \in U_{4 k+2-n}^{-n}(B)$. Of course, if $B^{4 k+1-n} \subset B$ denotes a $4 k+1-n-$ skeleton, we have $x_{\mid B^{4 k+1-n}} \in U_{\infty}^{-n}\left(B^{4 k+1-n}\right)$. We also have seen that $d_{4 k+1-n}^{-n}\left(x_{\mid B^{4 k+1-n}}\right)=d_{4 k+1-n}^{-n}(x)_{\mid B^{4 k+1-n}}$ determines $d_{4 k+1-n}^{-n}(x)$ uniquely. The interesting feature of the analytic definition is that it shows that $d_{4 k+1-n}^{-n}\left(x_{\mid B^{4 k+1-n}}\right)$ admits an extension from $B^{4 k+1-n}$ to $B$.

In the following Lemmas we give an alternative proof of this property.

4.6.2 Let $f: B \rightarrow \Omega^{n} B O$ be a map. Assume that the restriction $f^{r}:=f_{\mid B^{r}}: B^{r} \rightarrow$ $\Omega^{n} B O$ of $f$ to a $r$-skeleton $B^{r}$ of $B$ factors over a map $g^{r}: B^{r} \rightarrow \Omega^{n}(U / O)$ (ie $f^{r}=\Omega^{n} i \circ g^{r}$, where $i: U / O \rightarrow B O$ is as above). Assume further that $r+n \equiv 1$ $(\bmod 8)$. Let $R$ be some abelian group.

Lemma 4.11 If $x \in H^{k}\left(\Omega^{n} U / O, R\right)$ satisfies $2 x=0$, then the class $\left(g^{r}\right)^{*}(x)$ extends from $B^{r}$ to $B$.

Since the map $H^{r}(B ; R) \rightarrow H^{r}\left(B^{r} ; R\right)$ is injective, this extension is unique. Note that the Lemma in particular applies to the cohomology classes listed in Theorem 4.10. 
Proof Let $K(R ; r)$ denote the Eilenberg-Mac Lane space which represents the functor $H^{r}(\ldots, R)$. We represent the cohomology class $x$ by a map $x: \Omega^{n}(U / O) \rightarrow K(R ; r)$. It suffices to show that $\left(g^{r}\right)^{*}(x)$ extends to a $(r+1)$-skeleton $B^{r+1}$ of $B$ (such that $\left.B^{r} \subset B^{r+1}\right)$. In fact, it then further extends to $B$ since the inclusion $B^{r+1} \rightarrow B$ is a $(r+1)$-equivalence.

The universal example is given by the space $B=K^{r+1}$ which is obtained from $\Omega^{n}(U / O)$ by attaching $(r+1)$-cells in such a way as to kill the kernel of the map $\left(\Omega^{n} i\right)_{r}: \pi_{k} \Omega^{n}(U / O) \rightarrow \pi_{r}\left(\Omega^{n} B O\right)$. Here $f$ is obtained from $\Omega^{n} i: \Omega^{n}(U / O) \rightarrow$ $\Omega^{n} B O$, which extends to some map $f: K^{r+1} \rightarrow \Omega^{n} B O$ by the construction of $K^{r+1}$ (and elementary obstruction theory), and $g^{r}$ is the inclusion of the $r$-skeleton of $\Omega^{n}(U / O)$ (and therefore of $\left.K^{r+1}\right)$ into $\Omega^{n}(U / O)$.

In our case we have $\pi_{r}\left(\Omega^{n}(U / O)\right) \cong \mathbb{Z}$ and $\operatorname{ker}\left(\Omega^{n} i\right)_{r} \cong 2 \mathbb{Z}$ (as follows from Bott periodicity and the long exact homotopy sequence (in low degrees) of $U / O \rightarrow$ $B O \rightarrow B U)$. If $\phi: S^{r} \rightarrow \Omega^{n}(U / O)$ represents a generator of $\pi_{r}\left(\Omega^{n}(U / O)\right)$, and if $h: S^{r} \rightarrow \Omega^{n}(U / O)$ represents twice this generator, ie a generator of $\operatorname{ker}\left(\Omega^{n} i\right)_{r}$, then $h^{*}\left(g^{r}\right)^{*} x=2 \phi^{*}\left(g^{r}\right)^{*} x=0$. Thus the map $x \circ h$ is null homotopic, and therefore $\left(g^{r}\right)^{*} x$ extends to $K^{r+1}$.

4.6.3 Let still $f: B \rightarrow \Omega^{n} B O$ be a map and assume that the restriction $f^{4 k+1-n}=$ $f_{\mid B^{4 k+1-n}}: B^{4 k+1-n} \rightarrow \Omega^{n} B O$ of $f$ to a $4 k+1-n$-skeleton $B^{4 k+1-n}$ of $B$ factors over a map $g^{4 k+1-n}: B^{4 k+1-n} \rightarrow \Omega^{n}(U / O)$ (ie $f^{4 k+1-n}=\Omega^{n} i \circ g^{4 k+1-n}$, where $i: U / O \rightarrow B O$ is as above).

Lemma 4.12 If

$$
x=\left[\Omega^{n}\left(\frac{(m-1) !}{(2 k) !} T\left(c_{4 k+2}\right)\right)\right]_{\mathbb{Q} / \mathbb{Z}} \in H^{4 k-n+1}\left(\Omega^{n}(U / O)\right)
$$

is one of the classes of Theorem 4.10, then $\left(f^{4 k+1-n}\right)^{*} x$ extends from $B^{4 k+1-n}$ to $B$.

4.6.4 Note that half of the cases are already covered by Lemma 4.11, namely whenever the dimension condition is satisfied, ie when $(4 k+1-n)+n \equiv 1(\bmod 8)$, in other words, if $k$ is even.

4.6.5 Moreover, the cases $n \equiv 0(\bmod 8)$ as well as $n \equiv 1(\bmod 8)($ and $4 k+1=n)$ are trivial, because in these cases we have seen that the characteristic class $x$ already pulls back from $\Omega^{n} B O$ to $\Omega^{n}(U / O)$ : it is expressed in terms of Stiefel-Whitney classes in the first case, and in terms of the dimension of the bundle in the second case. 
4.6.6 We use the proof Lemma 4.11 to deal with the remaining cases. This proof shows that it suffices to treat the case $B^{4 k+1-n}=S^{4 k+1-n}$ and to show that the pullback class $\left(f^{4 k+1-n}\right)^{*} x$ vanishes for arbitrary $f: S^{4 k-n+1} \rightarrow \Omega^{n}(U / O)$ (and therefore extends over the disc $D^{4 k+2-n}$ ).

4.6.7 Observe that, by Equation (8), the cohomology class $x$ is obtained as pull back of $\frac{1}{2} c_{4 k+1-n}$ or $\frac{1}{2}(2 k-(n-1) / 2) ! \mathbf{c h}_{[4 k-(n-1)]}$ from $U$ or $B U$, respectively (depending on the parity of $n$ ). However, on all spheres the Chern character is integral, ie for an arbitrary map $f: S^{k} \rightarrow B U, f^{*} \mathbf{c h} \in H^{*}\left(S^{k} ; \mathbb{Z}\right)$.

4.6.8 If $n \equiv 7(\bmod 8)$ and $k$ odd $($ and $4 k>n)$ then $4 k+1-n \geq 4$. This implies $\frac{1}{2}(2 k-(n-1) / 2) ! \in \mathbb{Z}$. So the cohomology class $\frac{1}{2}(2 k-(n-1) / 2) ! \mathbf{c h}_{[4 k-(n-1)]}$ pulls back to 0 in $H^{*}\left(S^{4 k-(n-1)} ; \mathbb{Q} / \mathbb{Z}\right)$ for an arbitrary map $S^{4 k-(n-1)} \rightarrow B U$ by by 4.6.7. As observed in 4.6.4, $n \equiv 7(\bmod 8)$ and $k$ even is covered by Lemma 4.11.

4.6.9 For $n \equiv 6(\bmod 8)$ and an arbitrary map $f: S^{4 k-n+1} \rightarrow U$, by Lemma A.6 and Theorem B.4

$$
f^{*}\left(\frac{1}{2} c_{4 k+1-n}\right)=\Sigma^{-1} F^{*}\left(\frac{1}{2} \Omega c_{4 k+1-n}\right)=\Sigma^{-1}\left(F^{*} \frac{1}{2}\left(2 k-\frac{n}{2}\right) ! \mathbf{c h}_{[4 k-n]}\right) .
$$

Here, $\Sigma: H^{4 k-n}\left(S^{4 k-n}\right) \rightarrow H^{4 k-n+1}\left(S^{4 k-n+1}\right)$ is the suspension isomorphism and

$$
F: S^{4 k-n} \rightarrow \Omega U=B U \times \mathbb{Z}
$$

is the adjoint of $f: \Sigma S^{4 k-n}=S^{4 k-n+1} \rightarrow U$. Again, if $4 k-n \geq 4$ then $(2 k-n / 2) ! \in$ $2 \mathbb{Z}$ and therefore by 4.6.7 $F^{*}\left(\frac{1}{2}(2 k-n / 2) ! \mathbf{c h}_{[4 k-(n-1)]}=0\right.$. However, if $4 k-n=2$ then, since $n \equiv 6(\bmod 8) k$ is even and therefore $(4 k+1-n)+n \equiv 1(\bmod 8)$, such that this case is covered by Lemma 4.11.

4.6.10 If $n \equiv 2,3,4,5(\bmod 8)$, then $x=0$, which trivially extends. This concludes the proof of Lemma 4.12 .

\section{Appendix A Transgression}

\section{A.1 Transgression in cohomology}

A.1.1 In this section, we want to recall the general definition of transgression and its basic properties. Special cases are "suspension" or "looping". All of this is well known, and included here for the convenience of the reader.

A.1.2 The situation is the following: let $f: E \rightarrow B$ be a map, and $b \in B$ a point. Write $F_{b}:=f^{-1}(b)$. Let $i: F_{b} \rightarrow E$ be the inclusion. Let $H^{*}$ be any (generalized) cohomology theory. In the following, the loop spaces $\Omega B$ are defined with respect to the basepoint $b$. Suspensions are reduced suspensions. 
A.1.3 The adjoint of the identity map $\Omega B \rightarrow \Omega B$ gives a canonical map $\Sigma \Omega B \rightarrow B$. This induces $H^{*}(B) \rightarrow H^{*}(\Sigma \Omega B)$.

Definition A.1 We define the loop map

$$
\Omega: H^{*}(B) \rightarrow H^{*-1}(\Omega B) .
$$

as the composition of $H^{*}(B) \rightarrow H^{*}(\Sigma \Omega B)$ with the suspension isomorphism $H^{*}(\Sigma \Omega B) \rightarrow H^{*-1}(\Omega B)$.

By construction and functoriality of the suspension isomorphism, the loop map is functorial, too.

A.1.4 Given the map $f: E \rightarrow B$, consider the cofibration sequence $E \rightarrow Z f \rightarrow C f$, where $Z f$ is the mapping cylinder and $C f$ the mapping cone. The inclusion $B \rightarrow Z f$ is a homotopy equivalence. The long exact sequence in cohomology of this cofibration gives

$$
\begin{gathered}
H^{k}(B) \\
\| \\
\cdots \rightarrow H^{k}(C f) \rightarrow H^{k}(Z f) \stackrel{f^{*}}{\longrightarrow} H^{k}(E) \rightarrow \cdots
\end{gathered}
$$

In particular, $H^{k}(C f)$ maps surjectively onto $\operatorname{ker}\left(f^{*}\right) \subset H^{k}(B)$.

A.1.5 Consider now $\Omega C f$. Since the composition $F \stackrel{i}{\rightarrow} E \stackrel{f}{\rightarrow} B$ is the constant map to $b$, we can define a canonical map

$$
l: F \rightarrow \Omega C f, \quad x \mapsto c_{x},
$$

where $c_{x}$ is the loop in $C f$ with $c_{x}(0)=b \in B, c_{x}(t)=(x, t) \in E \times(0,1) \subset C f$, and $c_{x}(1)$ is the cone point in $C f$.

Mapping $B$ to the second cone point gives the second map $j$ in the cofibration sequence $B \rightarrow C f \stackrel{j}{\rightarrow} \Sigma E$. From this we conclude that the kernel of $H^{k}(C f) \rightarrow H^{k}(B)$ equals $\operatorname{im}\left(j^{*}\right)$. The composition $F \rightarrow \Omega C f \rightarrow \Omega \Sigma E$ can be factored as $F \stackrel{i}{\rightarrow} E \rightarrow \Omega \Sigma E$, where the second map is the adjoint of the identity map $\Sigma E \rightarrow \Sigma E$.

A.1.6 We now recall the definition of transgression.

Definition A.2 We define the transgression

$$
T: H^{k}(B) \supset \operatorname{ker}\left(f^{k}\right) \rightarrow H^{k-1}(F) / \operatorname{im}\left(i^{*}\right)
$$

as the composition

$$
\operatorname{ker}\left(f^{*}\right) \cong H^{k}(C f) / \operatorname{im}\left(j^{*}\right) \stackrel{\Omega}{\rightarrow} H^{k-1}(\Omega C f) / \operatorname{im}\left((\Omega j)^{*}\right) \rightarrow H^{k-1}(F) / \operatorname{im}\left(i^{*}\right) .
$$


Note that for the second map we used the factorization which shows that $\operatorname{im}\left((\Omega j)^{*}\right)$ goes into $\operatorname{im}\left(i^{*}\right)$.

It is clear from the construction that transgression is natural with respect to the map $f: E \rightarrow B$, ie given a diagram

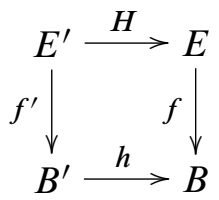

we have an equality of the form $T^{\prime} \circ h^{*}=H^{*} \circ T$.

Definition A.3 The elements of $\operatorname{ker}\left(f^{*}\right) \subset H^{*}(B)$ are called transgressive. These are the classes whose transgression is defined.

A.1.7 Next we relate transgression to the loop map and to suspension.

Lemma A.4 The transgression in the fibration $\Omega B \rightarrow P B \rightarrow B$, where $P B$ is the (contractible) space of paths ending at $b$ coincides with the loop map.

Proof Carry out the construction. If $f: P B \rightarrow B$ is the start point projection, use the homotopy equivalence $C f \rightarrow B$ which maps $(p, s) \in P B \times(0,1) \subset C f$ to $p(s)$ (recall that $s=1$ corresponds to the cone point).

Lemma A.5 The transgression of $B \hookrightarrow C B \rightarrow \Sigma B$ is the suspension isomorphism $\Sigma: H^{k+1}(\Sigma B) \rightarrow H^{k}(B)$.

Proof Carry out the construction. Use the "folding" homotopy equivalence $C f \rightarrow \Sigma B$ (where $f: C B \rightarrow \Sigma B$ is the projection). The composition of $\Sigma B \rightarrow C f$ with this homotopy equivalence is the identity map. Starting with $H^{*}(\Sigma B)$, we have to pull back with this map and then use the suspension isomorphism (by naturality of the latter).

A.1.8 Let $f: \Sigma X \rightarrow Y$ be a map with adjoint $F: X \rightarrow \Omega Y$. Then we have a commutative diagram of fibrations:

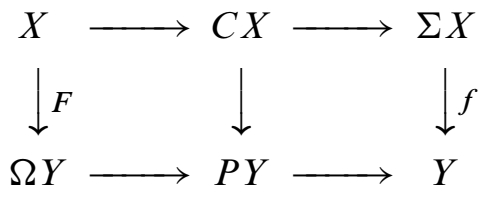

Algebraic 8 Geometric Topology, Volume 8 (2008) 
Lemma A.6 Then for each $a \in H^{*}(Y)$,

$$
F^{*}(\Omega a)=\Sigma f^{*} a .
$$

Proof This follows from naturality of transgression and the fact that both $\Omega$ and $\Sigma$ are transgression homomorphisms by Lemma A.4 and Lemma A.5.

A.1.9 In the construction of the transgression, we consider in particular the following commutative diagram of maps:

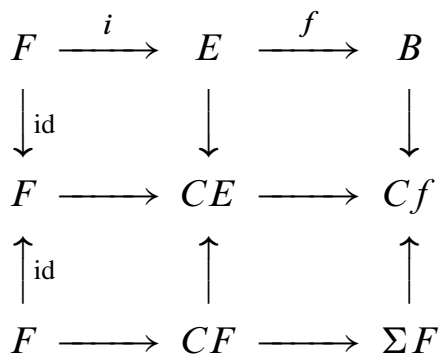

By naturality, the transgression homomorphism in $E \stackrel{f}{\rightarrow} B$ is determined by the transgression in $C F \rightarrow \Sigma F$ (this is of course, what we used in the construction), since $H^{*}(C f) \rightarrow H^{*}(B)$ surjects onto the transgressive classes.

A.1.10 We now prove the following lemma.

Lemma A.7 Let $\Phi: H^{*} \rightarrow h^{*}$ be a natural transformation between generalized cohomology theories. Transgression commutes with this natural transformation.

Proof Let $f: E \rightarrow B$ be a continuous map. First observe that by naturality $\Phi$ maps $\operatorname{ker}\left(H^{*}(f)\right)$ to $\operatorname{ker}\left(h^{*}(f)\right)$ and $\operatorname{im}\left(H^{*}(i)\right)$ to $\operatorname{im}\left(h^{*}(i)\right)$, so that the assertion makes sense.

The construction of the transgression homomorphism only uses maps induced from continuous maps between topological spaces (and their inverses) and the suspension isomorphism. By definition, a natural transformation between cohomology theories is compatible with such homomorphisms, and therefore also with transgression.

A.1.11 Let $F \stackrel{i}{\rightarrow} E \stackrel{f}{\rightarrow} B$ be a sequence of maps as above. This gives rise to the transgression homomorphism $T: H^{*}(B) \supset \operatorname{ker}\left(f^{*}\right) \rightarrow H^{*-1}(F) / \operatorname{im}\left(i^{*}\right)$.

Applying the loop space functor we also get the sequence of maps

$$
\Omega F \stackrel{\Omega i}{\longrightarrow} \Omega E \stackrel{\Omega f}{\longrightarrow} \Omega B,
$$


with associated transgression homomorphism

$$
T_{\Omega}: H^{*}(\Omega B) \supset \operatorname{ker}\left(\Omega f^{*}\right) \rightarrow H^{*-1}(\Omega F) / \operatorname{im}\left((\Omega i)^{*}\right) .
$$

Proposition A.8 If $x \in H^{n}(B)$ is transgressive, then

$$
\Omega T(x)=T_{\Omega}(\Omega x) \in H^{n-2}(\Omega F) / \operatorname{im}\left((\Omega i)^{*}\right) .
$$

Proof We obtain the following commutative diagram

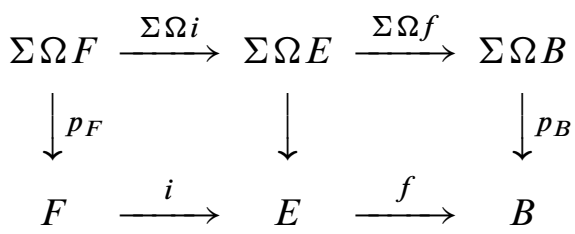

where the vertical maps are adjoints of the identity maps $\Omega \cdot \rightarrow \Omega \cdot$. Since we work with the reduced suspension, the inclusion $\Sigma \Omega F \hookrightarrow \Sigma \Omega E$ is the fiber of $\Sigma \Omega f$. By naturality of the transgression, $p_{F}^{*}(T x)=T_{\Sigma \Omega}\left(p_{B}^{*} x\right)$ for each transgressive class $x \in H^{*}(B)$. The suspension isomorphism maps by definition $p_{F}^{*}(T x)$ to $\Omega(T x)$ and $p_{B}^{*} x$ to $\Omega x$. By Lemma A.7 transgression commutes with the suspension isomorphism (indeed the suspension isomorphism can be interpreted as a natural transformation between cohomology theories). Therefore we have $\Omega(T x)=T_{\Omega}(\Omega x)$.

\section{A.2 Transgression and products}

A.2.1 Let $\Delta: B \rightarrow B \times B$ be the diagonal map. We still consider the map $f: E \rightarrow B$.

Definition A.9 (1) A class $x \in H^{*}(B)$ is called a nontrivial product, if $x=\Delta^{*} y$ for some $y \in H^{*}(B \times B)$ such that $\left(\operatorname{id}_{B} \times\{b\}\right)^{*} y=0=\left(\{b\} \times \mathrm{id}_{B}\right)^{*} y$.

(2) We say that the first factor of a nontrivial product $x$ is transgressive, if $x=\Delta^{*} y$ for an $y$ such that $\left(f \times \mathrm{id}_{B}\right)^{*} y=0 \in H^{*}(E \times B)$, similarly we define that the second factor is transgressive.

A.2.2 Note that if one of the factors of a product is transgressive, then so is the product.

Proposition A.10 The transgression of a nontrivial product with at least one transgressive factor is zero.

Algebraic 83 Geometric Topology, Volume 8 (2008) 
Proof This follows from naturality of the transgression. Consider the diagram

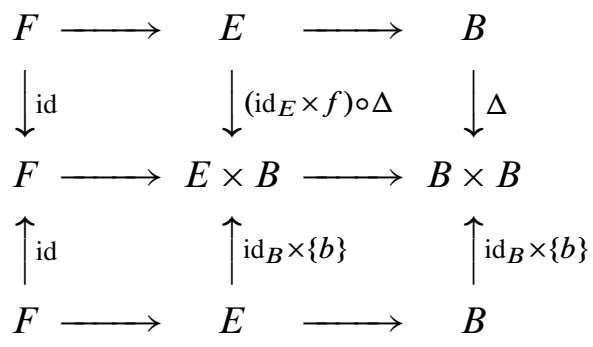

Let us assume that $x$ is transgressive in the first factor. By $T_{i}$ we denote the transgressions associated to the corresponding rows. Then we have $T_{1}(x)=T_{2}(y)=$ $T_{3}\left(\left(\operatorname{id}_{B} \times\{b\}\right)^{*} y\right)=0$, since $\left(\operatorname{id}_{B} \times\{b\}\right)^{*} y=0$.

A.2.3 Let us consider the following example. Define $N:=T^{2} \backslash\left(D^{2}\right)^{\circ}$, ie $N$ is the two torus with an open disc removed. Let $f: N \rightarrow T^{2}$ be the map which collapses the boundary of $N$ to one point. On the one hand, the fundamental class $\left[T^{2}\right] \in H^{2}\left(T^{2}, \mathbb{Z}\right)$ is transgressive. On the other hand, $\left[T^{2}\right]$ is a nontrivial product of 1 -dimensional cohomology classes, and none of the factors is transgressive.

Collapsing the complement of an open disc in $T^{2}$ to a point gives a degree 1 map $g: T^{2} \rightarrow S^{2}$. If we write $S^{2}=\Sigma S^{1}$, we then get a diagram:

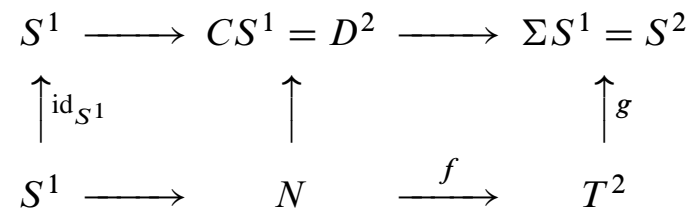

Here, $g^{*}\left[S^{2}\right]=\left[T^{2}\right]$, where $\left[S^{2}\right] \in H^{2}\left(S^{2}, \mathbb{Z}\right)$ is the fundamental class. By naturality, $T\left(\left[T^{2}\right]\right)=T\left(\left[S^{2}\right]\right)=\left[S^{1}\right] \in H^{1}\left(S^{1}, \mathbb{Z}\right)$ is the fundamental class of $S^{1}$, in particular nonzero.

This shows that at least one of the factors in Proposition A.10 has to be transgressive for the assertion to hold.

\section{A.3 Transgression in ordinary cohomology and the relation with the Bockstein}

A.3.1 We now want to describe how one can construct the transgression in ordinary singular cohomology with coefficients on the level of chains. Let $f: E \rightarrow B$ be a map with fiber $i: F \hookrightarrow E$ over $b \in B$. Let $R$ be an abelian group. Assume that $x \in H^{k}(B, R)$ is transgressive, ie we have $f^{*} x=0$. We choose a cocycle $c \in C^{k}(B, R)$ representing $x$. Then the cocycle $f^{*} c$ is a boundary, ie there exists 
a chain $c_{0} \in C^{k-1}(E, R)$ with $d c_{0}=f^{*} c$. The restriction of $c_{0}$ to $F$ is closed, since $d i^{*} c_{0}=i^{*} d c_{0}=i^{*} p^{*} c$. It follows that $i^{*} c_{0}$ represents a cohomology class $\left[i^{*} c_{0}\right] \in H^{k-1}(F, R)$. The cocycle $c_{0}$ is well defined only up to closed cocycles in $E$. It follows that the class $\left[c_{0}\right]$ is well defined only up to the image of $i^{*}$. Hence we get a well-defined class $\tilde{T}(x) \in H^{k-1}(F) / \operatorname{im}\left(i^{*}\right)$.

Proposition A.11 We have $\tilde{T}(x)=T(x)$.

Proof The recipe described in the proposition defines a transformation $\widetilde{T}$ which is again natural with respect to the map $f: E \rightarrow B$. As explained in the A.1.9 it must coincide with the transgression $T$ if it does so in the special case of the cofibration $B \rightarrow C B \rightarrow \Sigma B$. But in this case the above description produces exactly the suspension isomorphism which is by definition the transgression map $T$.

A.3.2 If $f: E \rightarrow B$ is a map of smooth manifolds and $R=\mathbb{R}$, then we could replace the singular cochains by differential forms and construct $\widetilde{T}$ on the level of forms. Again we get $\widetilde{T}=T$.

A.3.3 For a cohomology class $x \in H^{*}(X, \mathbb{Z})$ let $x^{\mathbb{Q}} \in H^{*}(X, \mathbb{Q})$ denote the image of $x$ under the canonical coefficient homomorphism $\mathbb{Z} \rightarrow \mathbb{Q}$. Let $n \in \mathbb{N}$ and $\beta$ be the Bockstein transformation associated to the sequence

$$
0 \rightarrow \mathbb{Z} \stackrel{\cdot n}{\rightarrow} \mathbb{Z} \rightarrow \mathbb{Z} / n \mathbb{Z} \rightarrow 0
$$

A.3.4 Let $x \in H^{k}(X, \mathbb{Z})$ be such that $n x$ is transgressive. Note that then $q^{\mathbb{Q}}$ is transgressive, too. Since $n f^{*} x=0$ by the Bockstein exact sequence there exist $u \in H^{k-1}(E, \mathbb{Z} / n \mathbb{Z})$ with $\beta(u)=f^{*} x$. Recall that $T(n x)$ is an equivalence class of cohomology classes.

Proposition A.12 We have

$$
\begin{gathered}
T(n x) \ni i^{*} u, \\
n T\left(x^{\mathbb{Q}}\right) \ni i^{*} u^{\mathbb{Q}} .
\end{gathered}
$$

Proof We use the description of the transgression on the singular cochain level given in Proposition A.11. Let $c$ be an integral cocycle representing $x$. Let $c_{0}$ be an integral cochain of $E$ with $d c_{0}=n f^{*} c$. Then $i^{*} c_{0}$ represents $T(n x)$.

The reduction of $c_{0}$ modulo $n \mathbb{Z}$ becomes closed and therefore represents a cohomology class $u \in H^{k-1}(E, \mathbb{Z} / n \mathbb{Z})$. By the explicit construction of the Bockstein homomorphism, $\beta(u)=f^{*} x$. Equation (12) follows.

Since transgression commutes with the passage to rational coefficients by Lemma A.7, Equation (13) follows from Equation (12). 


\section{Appendix B Cohomology of $B O, B U$ and their loop spaces}

\section{B.1 The cohomology}

B.1.1 In this appendix, we summarize the main results about the cohomology of $B O$, $B U$ and their loop spaces, and the relations between them, including the determination of the transgression homomorphisms. These results are all classical, and almost all of them can be found in Cartan's papers [8], where these calculations were essential in his cohomological proof of Bott periodicity. Since they are scattered over these papers, we collect them here in more convenient form. All results without a proof or a different reference can be found in [8].

B.1.2 Bott periodicity gives canonical (up to homotopy) homotopy equivalences between $\Omega^{n} B O$ and other classical spaces summarized in the following list.

\section{Theorem B.1}

\begin{tabular}{|c|c|c|c|c|c|c|c|c|c|}
\hline$n-1$ & -1 & 0 & 1 & 2 & 3 & 4 & 5 & 6 & 7 \\
\hline$\Omega^{n}(U / O)$ & $U / O$ & $B O \times \mathbb{Z}$ & $O$ & $O / U$ & $U / S_{I}$ & $3 S p \times$ & $S p$ & $S p / L$ & $U / O$ \\
\hline or & & & $\times \mathbb{Z}$ & $/ U \times 2$ & & & & & \\
\hline
\end{tabular}

This extends 8-periodically.

In the following, we will frequently identify the (cohomology of) different loop spaces of spaces in this table using the corresponding homotopy equivalence without further mentioning it. Note that we have done so already throughout the body of the paper.

B.1.3 In the following, $L\left(x_{i_{1}}, x_{i_{2}}, \ldots\right)$ denotes a polynomial algebra in the generators $x_{i}$, where by convention $x_{i}$ has (cohomological) degree $i$, and $E\left(y_{i_{1}}, y_{i_{2}}, \ldots\right)$ denotes an exterior algebra, with similar degree conventions for the generators.

B.1.4 In the following list, we describe the cohomology of the connected component of the base point in $\Omega^{k} B O$. Note that we "rename" some of the usual characteristic classes like the Pontryagin classes: $p_{4}$ is a cohomology class in $H^{4}$ etc. 


\section{Theorem B.2}

\begin{tabular}{ccccl}
$k$ & $\Omega^{k} B O$ & $H^{*}\left(\Omega^{k} B O_{0}, \mathbb{Z}\right)$ & $H^{*}\left(\Omega^{k} B O_{0}, \mathbb{Z} / 2 \mathbb{Z}\right)$ & $H^{*}\left(\Omega^{k} B O_{0}, \mathbb{Z}\left[\frac{1}{2}\right]\right)$ \\
\hline 0 & $B O$ & $L\left(p_{4}, p_{8}, \ldots\right) \oplus 2-$ Tors & $L\left(w_{1}, w_{2}, \ldots\right)$ & $L\left(p_{4}, p_{8}, \ldots\right)$ \\
1 & $O$ & $*$ & $L\left(d_{1}, d_{3}, \ldots\right)$ & $E\left(v_{3}, v_{7}, \ldots\right)$ \\
2 & $O / U$ & $L\left(u_{2}, u_{6}, \ldots\right)$ & & \\
3 & $U / S p$ & $E\left(a_{1}, a_{5}, \ldots\right)$ & & \\
4 & $B S p \times \mathbb{Z}$ & $L\left(y_{4}, y_{8}, \ldots\right)$ & & \\
5 & $S p$ & $E\left(y_{3}, y_{7}, \ldots\right)$ & & \\
6 & $S p / U$ & $L\left(u_{2}, u_{6}, \ldots\right)^{*}$ & $E\left(c_{2}, c_{4}, \ldots\right)$ & $L\left(c_{2}, \ldots\right)$ \\
6 & $S p / U$ & $\frac{L\left[c_{2}, c_{4}, \ldots\right]}{\sum_{i+j=2 k}(-1)^{i} c_{2 i} c_{2 j}}$ & & \\
7 & $U / O$ & $E\left(a_{4 k+1}\right) \oplus 2-$ Tors & $E\left(w_{1}, w_{2}, \ldots\right)$ & $E\left(a_{1}, a_{5}, \ldots\right)$
\end{tabular}

We add the following detailed explanations, using the description of the loop spaces as in Theorem B.1.

(1) (a) $H^{*}(B O ; \mathbb{Z})$ contains a subalgebra isomorphic to the quotient by its torsion.

(b) This is a polynomial algebra $L\left(p_{4}, p_{8}, \ldots\right)$.

(c) The torsion is annihilated by 2 , it is the image of Bockstein.

(d) Reduction mod 2 maps $p_{4 k}$ to $\left(w_{2 k}\right)^{2}$.

(e) The classes $w_{2 k+1} \in H^{2 k+1}(B O, \mathbb{Z} / 2 \mathbb{Z})$ have unique lifts to $H^{2 k+1}(B O, \mathbb{Z})$ which we also denote by $w_{2 k+1}$.

(f) The same is true for every class in degree $k$ for $k$ not divisible by 4 , since in these degrees $H^{k}(B O, \mathbb{Z})$ is annihilated by 2 .

(2) Most complicated is the cohomology of $S O$ with $\mathbb{Z}$-coefficients, for reasons of space simply denoted $*$ in the list (case $k=1$ ). We can say the following:

(a) The torsion in $H^{*}(S O, \mathbb{Z})$ is annihilated by 2, it is the image of Bockstein.

(b) The quotient of $H^{*}(S O, \mathbb{Z})$ by its torsion is an exterior algebra $E\left(\overline{v_{3}}, \overline{v_{7}}, \ldots\right)$. It does not split back to $H^{*}(S O, \mathbb{Z})$ because of the product structure (compare with $\left.H^{*}(S O, \mathbb{Z} / 2 \mathbb{Z})\right)$.

(c) But of course, each monomial $\overline{v_{i_{1}}} \ldots \overline{v_{i_{s}}}$ has an inverse image $v_{i_{1}} \ldots v_{i_{s}} \in$ $H^{*}(S O, \mathbb{Z})$ (only additive! no multiplicative structure) which is well defined up to torsion, and the products are correct up to torsion.

(3) The integral cohomology of $S p / U$ (case $k=6$ ) is the dual of $L\left(u_{2}, u_{6}, \ldots\right.$ ). This shows in particular, that it is torsion-free. As a ring, it is the quotient of $L\left(c_{2}, c_{4}, \ldots\right)$ by the ideal generated by the elements $\sum_{i+j=2 k}(-1)^{i} c_{2 i} c_{2 j}$.

(4) (a) $H^{*}(U / O, \mathbb{Z})$ contains a subalgebra isomorphic to the quotient by its torsion, this is an exterior algebra $E\left(a_{1}, a_{5}, \ldots\right)$.

(b) The torsion is annihilated by 2 , it is the image of Bockstein. 
(c) Reduction mod 2 maps $a_{4 k+1}$ to $w_{2 k} w_{2 k+1}+\beta\left(w_{4 k}+w_{2} w_{4 k-2}+\cdots+\right.$ $\left.w_{2 k-2} w_{2 k+2}\right)$.

B.1.5 We also need the complex case, ie $B U$ (and will later relate $B O$ to $B U$ ). The case of $B U$ is of course much easier because of 2-periodicity, and since the cohomology does not contain torsion.

\section{Theorem B.3}

\begin{tabular}{ccc}
$k$ & natural homotopy equivalence of $\Omega^{k} B U$ to & $H^{*}\left(\Omega^{k} B U_{0}, \mathbb{Z}\right)$, \\
\hline 0 & $B U$ & $L\left(c_{2}, c_{4}, \ldots\right)$ \\
1 & $U$ & $E\left(c_{1}, c_{3}, \ldots\right)$ \\
2 & $B U \times \mathbb{Z}$. &
\end{tabular}

B.1.6 We now describe the effect of the loop map $\Omega: H^{*}(X) \rightarrow H^{*-1}(\Omega X)$ for integral cohomology and some of the spaces in Theorem B.1.

For the following table, recall that the universal Chern character is a certain rational polynomial in the universal Chern classes, and we have a unique integral lift $k$ ! $\mathbf{c h}_{2 k} \in$ $H^{2 k}(B U \times \mathbb{Z} ; \mathbb{Z})$.

\section{Theorem B.4}

\begin{tabular}{cccc} 
Space $X$ & $\Omega X$ & $x \in H^{*}(X, \mathbb{Z})$ & $\Omega(x) \in H^{*-1}(\Omega X, \mathbb{Z})$ \\
\hline$B U$ & $U$ & $c_{2 k}$ & $c_{2 k-1}$ \\
$U$ & $B U \times \mathbb{Z}$ & $c_{2 k-1}$ & $(k-1) ! \mathbf{c h}_{2 k-2}$ \\
$B S p$ & $S p$ & $y_{4 k}$ & $y_{4 k-1}$ \\
$B O$ & $O$ & $p_{4 k}$ & $2 v_{4 k-1}+$ Tors
\end{tabular}

Proof We only have to prove that $\Omega\left(c_{2 k-1}\right)=(k-1) ! \mathbf{c h}_{k-2}$. For this, observe that

$$
c_{2 k-1}=\Omega\left(c_{2 k}\right)=\Omega\left((k-1) ! \mathbf{c h}_{2 k}\right)=(k-1) ! \Omega\left(\mathbf{c h}_{2 k}\right),
$$

since the other summands in $\mathbf{c h}_{2 k}$ are decomposable and because the loop map applied to a decomposable class is zero by Proposition A.10. Now the Chern character is compatible with Bott periodicity, and therefore $\Omega^{2}\left(\mathbf{c h}_{2 k}\right)=\mathbf{c h}_{2 k-2}$. Consequently

$$
\Omega c_{2 k-1}=(k-1) ! \Omega^{2}\left(\mathbf{c h}_{2 k}\right)=(k-1) ! \mathbf{c h}_{2 k-2} .
$$


B.1.7 Now consider the next lemma.

Lemma B.5 The natural map

$$
l_{*}: H^{4 k+1}(B O, \mathbb{Z} / 2 \mathbb{Z}) \rightarrow H^{4 k+1}(B O, \mathbb{Q} / \mathbb{Z})
$$

of Equation (5) factors through the image of the Bockstein homomorphism

$$
\beta: H^{4 k+1}(B O, \mathbb{Z} / 2 \mathbb{Z}) \rightarrow H^{4 k+2}(B O, \mathbb{Z}) .
$$

Proof We have the following map of long exact sequences:

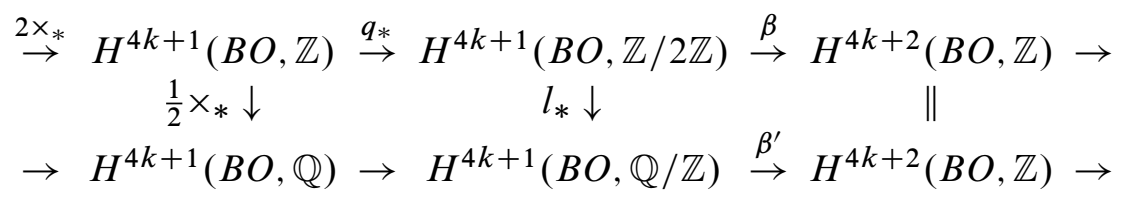

The assertion now follows from the fact that $H^{4 k+1}(B O, \mathbb{Q})=0$.

\section{B.2 Maps between loop spaces of $\mathrm{BO}$}

B.2.1 There is a large number of canonical maps between the different spaces in Theorem B.1 and in Theorem B.3 which are important for us and which are described in the following list:

(1) the inclusion $c: O \rightarrow U$ (given by complexification)

(2) the induced map $B c: B O \rightarrow B U$, which gives rise to $B c \times \operatorname{id}_{\mathbb{Z}}: B O \times \mathbb{Z} \rightarrow$ $B U \times \mathbb{Z}$

(3) the inclusion $q$ : $U \rightarrow S p$ (given by tensoring with the quaternions)

(4) the induced map $B q: B U \rightarrow B S p$

(5) the inclusion $f: U \rightarrow O$ (given by forgetting the complex structure)

(6) the induced map $B f: B U \rightarrow B O$

(7) the inclusion $j: S p \rightarrow U$ (given by forgetting the quaternionic structure)

(8) the induced map $B j: B S p \rightarrow B U$, which gives rise to $B j \times \operatorname{id}_{\mathbb{Z}}: B S p \times \mathbb{Z} \rightarrow$ $B U \times \mathbb{Z}$

(9) the projection $p: U \rightarrow U / O$

(10) the projection $P: U \rightarrow U / S p$

(11) the inclusion of the fiber $i: U / O \rightarrow B O$ obtained by dividing the total space of the universal principal $U$-fibration $U \rightarrow E U \rightarrow B U$ by $O$ (here we use the fact that $E U / O$ is a model for $B O$ ) 
(12) the fibration $B O \rightarrow B U$ constructed in (11)

(13) the map $\Omega_{i}^{7}: S p / U \rightarrow U / O$ obtained by looping this fibration seven times, it is the fiber of the map $U / O \rightarrow B O$ obtained by looping the fibration $B O \rightarrow B U$ seven times

(14) the (similar) inclusion of the fiber $I: S p / U \rightarrow B U$

(15) the (similar) inclusion of the fiber $\phi: O / U \rightarrow B U$

(16) a map $\alpha: U / O \rightarrow U$ given as composition

$$
U / O \stackrel{\sim}{\longrightarrow} \Omega S p / U \stackrel{\Omega I}{\longrightarrow} \Omega B U \stackrel{\sim}{\longrightarrow} U
$$

where the first map is the Bott periodicity homotopy equivalence, and the third is the usual homotopy equivalence (which is also part of (complex) Bott periodicity)

(17) a similar map $\beta: U / S p \rightarrow U$, given as composition

$$
U / S p \stackrel{\sim}{\longrightarrow} \Omega O / U \stackrel{\Omega \phi}{\longrightarrow} \Omega B U \stackrel{\sim}{\longrightarrow} U
$$

B.2.2 The following relations hold between these maps. As usual, we will freely use the Bott periodicity homotopy equivalences of Theorem B.1 and Theorem B.3 to identify certain loop spaces with other spaces (therefore, strictly speaking, the following assertions are true up to homotopy).

(1) It is a general fact in the theory of classifying spaces that one way to construct $B c$ in (2) is the fibration map of (12), which therefore can be identified with $B c$. Reason: the identity map $E U \rightarrow E U$, where the domain is considered as contractible $O$-principle bundle and the target as contractible $U$-principle bundle intertwines, using the inclusion $c: O \rightarrow U$ the structures as principle bundles. Therefore the induced map on the quotients is the map $B c$.

(2) The map (1) is obtained from (2) by applying the loop space transformation (and using the Bott periodicity identifications of $\Omega B O \stackrel{\sim}{\longrightarrow} O$ and $\Omega B U \stackrel{\sim}{\longrightarrow} U$ ).

(3) Similarly, (7) is obtained by applying the loop space functor to (8).

(4) By construction, looping (14) gives (16).

(5) By construction, looping (15) gives (17).

(6) Cartan [8] proves that $O / U \rightarrow B U$, ie (15) is obtained by applying the loop space functor to the inclusion (1), $O \rightarrow U$.

(7) Cartan [8] proves that looping (7) gives (14). This requires to check that his explicitly given maps $S p / U \rightarrow B U$ and $O / U \rightarrow B U$ are the fiber inclusions we claim they are. 
(8) Cartan [8] also checks that looping (17) gives (8).

(9) Cartan [8] proves that looping (16) gives (2). Strictly speaking, in this and the previous case he considers the corresponding maps of universal coverings $S U / S p \rightarrow S U$ which loops to $B S p \rightarrow B U$, and $S O / S U \rightarrow S O$ which loops to $B O \rightarrow B U$. Since we know that $U / S p \rightarrow U$ and $O / U \rightarrow O$ induce isomorphisms on $\pi_{1}$-level (all isomorphic to $\mathbb{Z}$ ), the claim follows.

B.2.3 To conclude, we have shown that in the sequence

$$
\begin{aligned}
B c \times \text { id: } & B O \times \mathbb{Z} \rightarrow B U \times \mathbb{Z} \\
c: & O \rightarrow U \\
\phi: & O / U \rightarrow B U \\
\beta & : U / S p \rightarrow U \\
B j \times \text { id: } & B S p \times \mathbb{Z} \rightarrow B U \times \mathbb{Z} \\
j & : S p \rightarrow U \\
I: & S p / U \rightarrow B U \\
\alpha: & U / O \rightarrow U \\
B c \times \text { id: } & B O \times \mathbb{Z} \rightarrow B U \times \mathbb{Z}
\end{aligned}
$$

each map is obtained by looping the previous one (and applying Bott periodicity to identify the loop spaces with the next spaces in the list).

B.2.4 In Table 1, we list the effect of the maps in cohomology. Again, this is due to Cartan [8], with a few exceptions easily obtained from his work. In these cases, the reason is indicated in the last column of Table 1. Recall that we always only consider the cohomology of the connected component of the base point. "By looping" means that we know that certain maps are obtained from each other by applying the loop space functor (and some canonical homotopy equivalences), and that we know the effect of the natural loop map functor $\Omega: H^{*}(X) \rightarrow H^{*-1}(\Omega X)$ by Theorem B.4.

B.2.5 In two cases, we have to take the different components into account: note that $\mathbf{c h}_{0} \in H^{0}(B U \times \mathbb{Z} ; \mathbb{Z})$ has the value $d$ (times the canonical generator) on the component of $B U \times \mathbb{Z}$ labeled by $d \in \mathbb{Z}$. Correspondingly, we have a real version $\mathbf{c h}_{0}^{\mathbb{R}} \in H^{0}(B O \times \mathbb{Z} ; \mathbb{Z})$ and a quaternionic version $\mathbf{c h}_{0}^{\mathbb{H}} \in H^{0}(B s p \times \mathbb{Z} ; \mathbb{Z})$, describing the dimension of the virtual universal real or quaternionic bundle, respectively. For these classes we get the (obvious) following relations under the maps induced by "complexification" or "forgetting the quaternionic structure", respectively. See Table 2 


\begin{tabular}{|c|c|c|c|}
\hline$f: X \rightarrow Y$ & $x \in H^{*}(Y, \mathbb{Z})$ & $f^{*}\left(x_{0}\right) \in H^{*}\left(X_{0}, \mathbb{Z}\right)$ & reason \\
\hline \multirow[t]{2}{*}{$B S p \rightarrow B U$} & $c_{4 k}$ & $y_{4 k}$ & \\
\hline & $c_{4 k+2}$ & 0 & \\
\hline \multirow[t]{2}{*}{$O / U \rightarrow B U$} & $c_{4 k+2}$ & $2 u_{4 k+2}$ & \\
\hline & $c_{4 k}$ & $-2 \cdot \sum_{0<i<2 k}(-1)^{i} f^{*}$ & $\left(c_{2 i}\right) / 2 \cdot f^{*}\left(c_{4 k-2 i}\right) / 2$ \\
\hline \multirow[t]{2}{*}{$S p / U \rightarrow B U$} & $c_{2 k}$ & $\overline{c_{2 k}}$ & \\
\hline & $c_{2 k} \bmod 2$ & $c_{2 k}$ & \\
\hline \multirow[t]{2}{*}{$S p \rightarrow U$} & $c_{4 k+1}$ & 0 & by looping \\
\hline & $c_{4 k+3}$ & $y_{4 k+3}$ & by looping \\
\hline \multirow[t]{2}{*}{$U / S p \rightarrow U$} & $c_{4 k+1}$ & $2 a_{4 k+1}$ & true dually in homology \\
\hline & $c_{4 k+3}$ & 0 & true dually in homology \\
\hline$U \rightarrow S p$ & $y_{4 k-1}$ & $2 c_{4 k-1}$ & $\begin{array}{l}\text { by looping (since prod- } \\
\text { ucts suspend to zero) }\end{array}$ \\
\hline \multirow{3}{*}{$\begin{array}{l}B U \rightarrow B S p \\
S O \rightarrow U\end{array}$} & $y_{4 k}$ & $\sum_{i+j=2 k}(-1)^{i} c_{2 i} c_{2 j}$ & \\
\hline & $c_{4 k+3}$ & $2 v_{4 k+3}+2-$ Tor & \\
\hline & $c_{4 k+1}$ & $2-$ Tor & \\
\hline \multirow{3}{*}{$\begin{array}{l}U \rightarrow U / O \\
U / O \rightarrow U\end{array}$} & $a_{4 k+1}$ & $2 c_{4 k+1}$ & \\
\hline & $c_{4 k+1}$ & $a_{4 k+1}+$ Tors & \\
\hline & $c_{4 k+3}$ & Tors & \\
\hline \multirow{4}{*}{$\begin{array}{l}U \rightarrow O \\
B O \rightarrow B U\end{array}$} & $v_{4 k+3}$ & $c_{4 k+3}$ & \\
\hline & $c_{4 k}$ & $p_{4 k}$ & \\
\hline & $c_{4 k+2}$ & $w_{2 k+1}^{2}$ & \\
\hline & $c_{4 k} \bmod 2$ & $w_{2 k}^{2}$ & \\
\hline \multirow[t]{3}{*}{$B U \rightarrow B O$} & $p_{4 k}$ & $\sum_{i+j=k}^{2 \kappa}(-1)^{i} c_{2 i} c_{2 j}$ & \\
\hline & $w_{2 k+1}$ & 0 & \\
\hline & $w_{2 k}$ & $c_{2 k} \bmod 2$ & \\
\hline \multirow[t]{2}{*}{$U / O \rightarrow B O$} & $w_{k} \bmod 2$ & $w_{k} \bmod 2$ & \\
\hline & $p_{4 k}$ & 0 & $\begin{array}{l}\text { mod } 2 \text { it maps to } 0 \text {, and } \\
\text { pulled back further to } \\
U \text { it is also } 0 \text {, ie no } 2- \\
\text { torsion and no free part }\end{array}$ \\
\hline
\end{tabular}

Table 1

\begin{tabular}{lll}
$f: X \rightarrow Y$ & $x \in H^{0}(Y, \mathbb{Q})$ & $f^{*}(x) \in H^{0}(X, \mathbb{Q})$ \\
\hline$B S p \times \mathbb{Z} \rightarrow B U \times \mathbb{Z}$ & $\mathbf{c h}_{0}$ & $2 \mathbf{c h}_{[0]}^{\mathbb{H}}$ \\
$B O \times \mathbb{Z} \rightarrow B U \times \mathbb{Z}$ & $\mathbf{c h}_{0}$ & $\mathbf{c h}_{[0]}^{\mathbb{R}}$
\end{tabular}

Table 2 
B.2.6 We conclude with two lemmas.

Lemma B.6 In the fibration

$$
U / O \stackrel{i}{\rightarrow} B O \stackrel{B c}{\longrightarrow} B U,
$$

the classes $c_{4 k+2}^{\mathbb{Q}} \in H^{4 k+2}(B U ; \mathbb{Q})$ are transgressive.

Proof The pull back of $c_{4 k+2}$ to $B O$ is 2-torsion in integral cohomology, therefore vanishes in rational cohomology.

Lemma B.7 In the fibration $S p / U \stackrel{\Omega^{7} i}{\longrightarrow} U / O \stackrel{\alpha}{\rightarrow} U$, the Leray-Serre spectral sequence for $H^{*}(\cdot, \mathbb{Z} / 2 \mathbb{Z})$ collapses at the $E_{2}$-term. In particular, the edge homomorphism

$$
\left(\Omega^{7} i\right)^{*}: H^{*}(U / O ; \mathbb{Z} / 2 \mathbb{Z}) \rightarrow H^{*}(S p / U ; \mathbb{Z} / 2 \mathbb{Z})
$$

is surjective, whereas the edge homomorphism

$$
\alpha^{*}: H^{*}(U ; \mathbb{Z} / 2 \mathbb{Z}) \hookrightarrow H^{*}(U / O ; \mathbb{Z} / 2 \mathbb{Z})
$$

is injective.

Proof $E_{2}=H^{*}(U / O ; \mathbb{Z} / 2 \mathbb{Z}) \otimes H^{*}(S p / U ; \mathbb{Z} / 2 \mathbb{Z})$ is the tensor product of an exterior algebra over $\mathbb{Z} / 2 \mathbb{Z}$ with exactly one generator in each positive even degree with an exterior algebra over $\mathbb{Z} / 2$ with one generator in each positive odd degree, ie an exterior algebra over $\mathbb{Z} / 2 \mathbb{Z}$ with one generator in each positive degree. It converges to an exterior algebra over $\mathbb{Z} / 2 \mathbb{Z}$ with one generator in each positive degree. Any nonzero differential would result in an $E_{\infty}$-term which is too low dimensional, therefore the spectral sequence necessarily collapses. The statement about the edge homomorphisms is an immediate consequence.

\section{References}

[1] MF Atiyah, F Hirzebruch, Vector bundles and homogeneous spaces, from: "Proc. Sympos. Pure Math., Vol. III”, Amer. Math. Soc. (1961) 7-38 MR0139181

[2] M F Atiyah, V K Patodi, I M Singer, Spectral asymmetry and Riemannian geometry. I, Math. Proc. Cambridge Philos. Soc. 77 (1975) 43-69 MR0397797

[3] MF Atiyah, I M Singer, Index theory for skew-adjoint Fredholm operators, Inst. Hautes Études Sci. Publ. Math. (1969) 5-26 MR0285033

[4] N Berline, E Getzler, M Vergne, Heat kernels and Dirac operators, Grundlehren series 298, Springer, Berlin (1992) MR1215720 
[5] J-M Bismut, J Cheeger, $\eta$-invariants and their adiabatic limits, J. Amer. Math. Soc. 2 (1989) 33-70 MR966608

[6] U Bunke, $\eta$-form, index theory and Deligne cohomology, to appear in Mem. Amer. Math. Soc. arXiv:math.DG/0201112

[7] U Bunke, X Ma, Index and secondary index theory for flat bundles with duality, from: "Aspects of boundary problems in analysis and geometry", Oper. Theory Adv. Appl. 151, Birkhäuser, Basel (2004) 265-341 MR2072502

[8] H Cartan, Démonstration homologique des théorèmes de périodicité de Bott. I, II, III, Périodicité des groupes d'homotopie stables des groupes, Sém. H. Cartan 12 16/17/18 (1959/60/61) MR0157863

[9] J Cheeger, J Simons, Differential characters and geometric invariants, from: "Geometry and topology (College Park, Md., 1983/84)", Lecture Notes in Math. 1167, Springer, Berlin (1985) 50-80 MR827262

[10] N Hitchin, Harmonic spinors, Advances in Math. 14 (1974) 1-55 MR0358873

[11] M Karoubi, K-theory, Grundlehren series 226, Springer, Berlin (1978) MR0488029

[12] J W Milnor, J D Stasheff, Characteristic classes, Annals of Math. Studies 76, Princeton University Press (1974) MR0440554

Universität Regensburg, Mathematische Fakultät

93040 Regensburg, Germany

Georg-August-Universität Göttingen, Mathematisches Institut

Bunsenstr. 3, 37073 Göttingen, Germany

bunke@uni-math.gwdg.de, schick@uni-math.gwdg.de

http://www . uni-regensburg.de/Fakultaeten/nat_Fak_I/Bunke,

www . uni-math.gwdg.de/schick

Received: 12 July 2005 Revised: 23 May 2008

Algebraic 83 Geometric Topology, Volume 8 (2008) 